\title{
Design and Analysis of a Reconfigurable Parallel Mechanism for Multidirectional Additive Manufacturing
}

\author{
Wei Ye, Yuefa Fang*, Sheng Guo \\ School of Mechanical, Electronic and Control Engineering, Beijing Jiaotong University, \\ Beijing 100044, P.R. China \\ Email: yffang@bjtu.edu.cn
}

\begin{abstract}
Reconfigurable parallel mechanisms (RPMs) have the advantages of high stiffness, good dynamic performance due to their parallel structures, and can reconfigure themselves into various mobility configurations to accommodate different task requirements, which indicate that RPMs have the potential to be used in multidirectional additive manufacturing. Based on this application, this paper focuses on the design and analysis of a novel RPM. Degree of freedom (DOF) requirements in different stages of multidirectional additive manufacturing are analyzed. A hybrid limb that is composed of a planar metamorphic mechanism and a 4-DOF serial chain is presented, and then employed in the design of a three-legged RPM. Unified model for kinematic position analysis and the overall Jacobian matrix of the RPM are established. Singularity analysis including inverse kinematic singularity and direct kinematic singularity analysis are conducted. Workspaces of the RPM in different operation modes are evaluated. A prototype and some experiments are presented.
\end{abstract}

KEYWORDS: Reconfigurable parallel mechanism, multidirectional additive manufacturing, kinematic position analysis, Jacobian matrix, singularity analysis

\section{Introduction}

Due to their parallel structures and reconfigurability, RPMs have the advantages of high stiffness, good dynamic performance, and can reconfigure themselves into various mobility configurations to accommodate different task requirements. Since the proposal in 1990s, RPMs have gained more and more interests in the research community. The initially proposed RPMs are ones that utilize modularity in the mechanisms [1, 2], whose reconfigurations are realized by attaching/detaching some modular components. These processes needing manual intervention are hard to accomplish during operation. Recently, four classes of RPMs that can realize reconfiguration without attaching/detaching modular parts are developed. The first class can be called more specifically as kinematotropic parallel mechanisms [3-5]. Reconfigurations of this kind of mechanisms occur in singular configurations called constraint singularity or C-space singularity [6, 7]. It should be noted that some mechanisms with different

* Corresponding author (email: yffang@bjtu.edu.cn) 
terminologies also belong to this class, such as parallel mechanisms that can change their group of motion [8], parallel mechanisms with multiple operation modes [9, 10], and parallel mechanisms with bifurcation of Schönflies motion [11]. The second class is metamorphic parallel mechanism [12-16], whose mobility variations are realized by altering some metamorphic joints or linkages into different working phases. The third class is parallel mechanism that employs lockable joints [17-20]. RPMs classified into the fourth class are ones with redundant kinematic joints [21, 22], which are equipped with motors and used to reconfigure the mechanisms into configurations with different dimensions to achieve optimal kinematical performance.

Except for the structural design aspect, performance analysis and application research of RPMs have also gained certain interests. Brisan et.al [23] presented the workspace and singularity analysis of a reconfigurable parallel robot system. Moosavian et.al [24] introduced a new approach to enhance the stiffness and static performance of RPMs without actuation redundancy. Chen [25] presented a method to identify the optimal configuration of a RPM that has the maximum dynamic load-carrying capacity. Yooh et.al [26] proposed a reconfigurable parallel robot for ankle rehabilitation that allows various exercise modes.

Parallel mechanisms have been already applied in several manufacturing fields such as in robot painting, laser welding and multi-axis kinematic machine. As a special manufacturing method that have attracted significant interests in recent years, the technique of additive manufacturing (AM) is to slice a 3D model into several 2D layers and then build the model layer by layer resorting to the translational motion of an extruder with respect to the workbench. The layer-based AM process with only translational motion has the drawback of stair-stepping effect [27, 28]. Furthermore, interference between the tool and the workpiece [29] would probably occur when adding materials on existed models such as in part reparation. To solve these problems, multidirectional AM technique that can add materials along multiple directions was developed. Several kinematic machines with serial structures [29-34] for multidirectional AM process were proposed. Compared with their serial counterparts, parallel mechanisms have several remarkable advantages such as higher stiffness, operation velocity, acceleration, and better dynamic performance. These advantages lead to the development of AM systems in parallel structures. Recently, a Delta robot based parallel kinematic machine [35] that has three translational motions was proposed for constant directional AM process. A low cost parallel kinematic machine based on Stewart platform [36] was developed for multidirectional AM process. It should be noted that in multidirectional AM processes, there will be various stages with different DOF requirements. For instance, 3T1R (three translational and one rotational) DOFs are required when adding materials on a cylindrical surface, only $3 \mathrm{~T}$ DOFs are needed when operating on a horizontal plane. However, due to the intercoupling characteristic, all the six inputs in the Stewart type multidirectional AM system should be collaborated together even though simple output motions such as 3-DOF translational motions are desired. The number of working inputs will be higher than that of the required DOFs, which goes against the concepts of energy saving and easy control. 
RPMs have the ability to reconfigure their structures into different mobility configurations with different number of working inputs, which indicates that the RPMs are suitable to be applied in multidirectional AM process. Based on this application, this paper focuses its attention on the design and analysis of a novel RPM. The main contents are as follows: section 2 conducts the DOF requirement analysis in different stages of multidirectional AM process and presents the design of a novel three-legged RPM. In section 3, unified model for kinematic position analysis is established. Section 4 builds the overall Jacobian matrix and section 5 conducts the singularity analysis. The workspaces of the RPM in different operation modes are evaluated and a prototype is presented in section 6. Finally, conclusions are drawn.

\section{Design and description}

First of all, the design objective is given, the desired multidirectional AM system is expected to have the ability of adding materials on various surfaces including free-form surface, spherical surface, cylindrical surface and horizontal surface, which means that the RPM should have different output DOFs to satisfy different task requirements. It is obvious that 3T2R DOFs are required while operating on a free-from surface or a spherical surface. $3 \mathrm{~T} 1 \mathrm{R}_{x}$ and $3 \mathrm{~T} 1 \mathrm{R}_{y}$ (the subscripts denote the rotational axes) DOFs are required respectively if the operation surfaces are cylindrical surfaces $A$ and $B$ as in Fig.1. Only 3T DOFs are required if materials are added on a horizontal plane. Therefore, the desired RPM should have four operation modes, i.e. 3T2R operation mode, $3 \mathrm{~T} 1 \mathrm{R}_{x}$ operation mode, $3 \mathrm{~T} 1 \mathrm{R}_{\mathrm{y}}$ operation mode and $3 \mathrm{~T}$ operation mode.

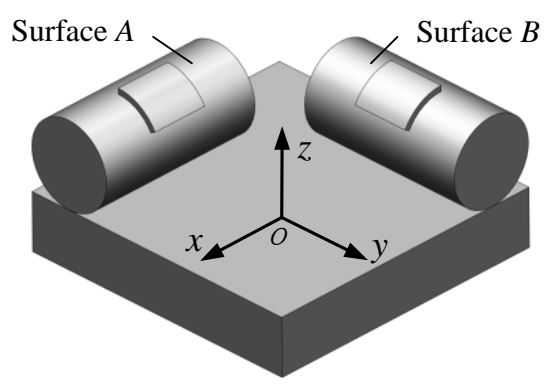

Fig.1 Two kinds of surface

In accordance with the aforementioned DOF requirements, a RPM for multidirectional AM is going to be designed. Since the minimum number of DOFs is 3, it is preferable to connect the moving platform and the fixed base with three limbs. Furthermore, the mechanism has at most 3T2R DOFs, there should exist one limb that generates a constraint couple on the moving platform. In view of the overall DOF requirements, the left two limbs should be reconfigurable and each has two configurations, in which one has six DOFs and the other one generates a constraint couple. Limb structure that generates a constraint couple (C-limb) can be chosen from our previous literature [37]. On the other hand, the reconfigurable limb that has six DOFs in one configuration and exerts a constraint couple in the other configuration should be designed. It has been demonstrated that reconfigurable closed chains including kinematotropic chains and planar metamorphic mechanisms can be used in the construction of reconfigurable hybrid limbs $[5,38]$. According to the constraint description, a hybrid PMM-URR(PMM represents a planar metamorphic 
mechanism, $\mathrm{U}$ represents a universal joint, R represents a revolute joint) limb is proposed as in Fig.2. In the reconfigurable hybrid limb, the axes of revolute joints $\mathrm{R}_{5}$ and $\mathrm{R}_{6}$ are parallel to the second rotational axis of universal joint $\mathrm{U}$, the first rotational axis of the universal joint is parallel to vector $\mathbf{P}_{2} \mathbf{P}_{3}\left(P_{2}\right.$ and $P_{3}$ are center points of two revolute joins in the PMM).

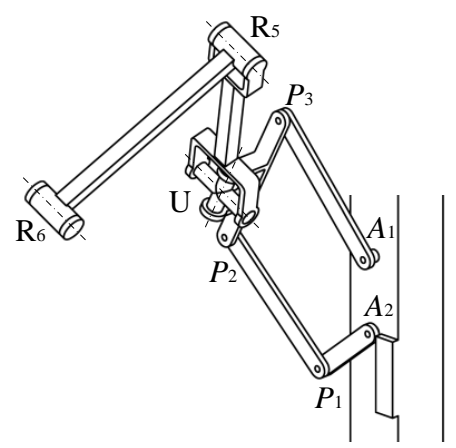

Fig. 2 A reconfigurable hybrid limb

The PMM integrated in the hybrid limb is a reconfigurable closed chain whose two employed phases are sketched as in Fig.3, the dimensional parameters of the PMM are specified as

$$
\left|P_{1} P_{2}\right|=\left|P_{2} P_{3}\right|=\left|A_{1} P_{3}\right|=2\left|A_{1} A_{2}\right|=2\left|A_{2} P_{1}\right|=l
$$

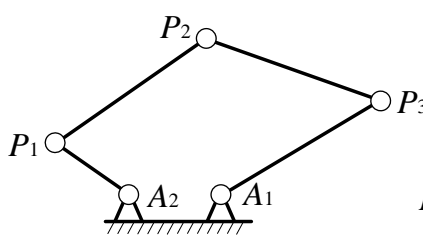

(a)

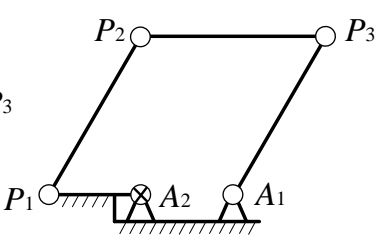

(b)

Fig.3 Phase (a) and phase (b) of the planar metamorphic mechanism

In a general configuration as in Fig.3(a), The output link $P_{2} P_{3}$ has two DOFs with active joints $A_{1}$ and $A_{2}$. When the mechanism moves to the position in Fig.3(b), link $A_{2} P_{1}$ is annexed with link $A_{1} A_{2}$ and joint $A_{2}$ is locked, length of the resulted link $A_{1} P_{1}$ is $l$, therefore, the mechanism degenerates into a planar four-bar parallelogram. The output link has a circular translational motion that is actuated by the motor mounted to joint $A_{1}$ in this configuration. The mechanism configurations in Fig.3(a) and Fig.3(b) are denoted by phase (a) and phase (b) hereafter.

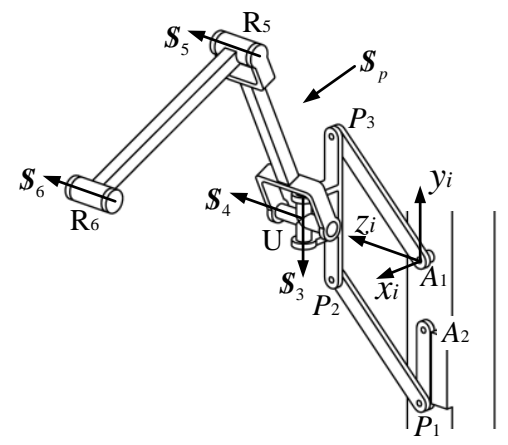

Fig.4 Hybrid limb with PMM in phase (b) 
The reconfigurable hybrid limb has two configurations associated with two phases of the PMM. One of the two configurations is sketched in Fig.2. With the PMM evolving into phase (b), the hybrid limb translates into the other configuration as in Fig.4.

It is obvious that with the PMM in phase (a), the hybrid limb has six DOFs and no constraint is exerted. However, when the PMM evolves into phase (b), the hybrid limb has only five DOFs, a constraint will be exerted on the body connected to this limb. Screw theory that has been widely used in constraint analysis of reconfigurable parallel mechanisms [13-16] will be employed here.Attach a local coordinate system $\left\{x_{i} y_{i} z_{i}\right\}$ to the hybrid limb with its original point located at the center of joint $A_{1}$ as in Fig. 4 . The $x_{i}$-axis is aligned with axis of joint $A_{1}$ and $y_{i}$-axis is aligned with line $A_{2} A_{1}$, the $z_{i}$ axis is defined according to the right-hand rule. The motion screw system $\left\{{ }^{i} \boldsymbol{S}\right\}$ of the hybrid limb expressed in local coordinate system $\left\{x_{i} y_{i} z_{i}\right\}$ is given by

$$
\left\{{ }^{i} \boldsymbol{S}\right\}=\left\{\begin{array}{l}
{ }^{i} \boldsymbol{S}_{\mathrm{p}}=\left[\begin{array}{llllll}
0 & 0 & 0 & 0 & y_{\mathrm{p}} & z_{\mathrm{p}}
\end{array}\right]^{\mathrm{T}} \\
{ }^{i} \boldsymbol{S}_{3}=\left[\begin{array}{llllll}
0 & 1 & 0 & -z_{3} & 0 & x_{3}
\end{array}\right]^{\mathrm{T}} \\
{ }^{i} \boldsymbol{S}_{4}=\left[\begin{array}{llllll}
g_{4} & 0 & h_{4} & y_{3} h_{4} & z_{3} g_{4}-x_{3} h_{4}-y_{3} g_{4}
\end{array}\right]^{\mathrm{T}} \\
{ }^{i} \boldsymbol{S}_{5}=\left[\begin{array}{llllll}
g_{4} & 0 & h_{4} & y_{5} h_{4} & z_{5} g_{4}-x_{5} h_{4}-y_{5} g_{4}
\end{array}\right]^{\mathrm{T}} \\
{ }^{i} \boldsymbol{S}_{6}=\left[\begin{array}{llllll}
g_{4} & 0 & h_{4} & y_{6} h_{4} & z_{6} g_{4}-x_{6} h_{4}-y_{6} g_{4}
\end{array}\right]^{\mathrm{T}}
\end{array}\right.
$$

where ${ }^{i} \boldsymbol{S}_{\mathrm{p}}$ represents the equivalent motion screw of the PMM in phase (b), ${ }^{i} \boldsymbol{S}_{3}$ and ${ }^{i} \boldsymbol{S}_{4}$ are motion screws of the universal joint, ${ }^{i} \boldsymbol{S}_{5}$ is the motion screw of revolute joint $\mathrm{R}_{5},{ }^{i} \boldsymbol{S}_{6}$ is the motion screw of revolute joint $\mathrm{R}_{6} .\left[\begin{array}{lllll}0 & y_{\mathrm{p}} & z_{\mathrm{p}}\end{array}\right]^{\mathrm{T}}$ is a unit vector that represents the direction of the equivalent motion screw ${ }^{i} S_{\mathrm{p}},\left[\begin{array}{lll}g_{4} & 0 & h_{4}\end{array}\right]^{\mathrm{T}}$ is a unit vector pointing in the direction of screws ${ }^{i} \boldsymbol{S}_{4},{ }^{i} \boldsymbol{S}_{5}$ and ${ }^{i} \boldsymbol{S}_{6} .\left[\begin{array}{lll}x_{3} & y_{3} & z_{3}\end{array}\right]^{\mathrm{T}}$ is the position vector of the center of the universal joint, $\left[\begin{array}{llllll}x_{5} & y_{5} & z_{5}\end{array}\right]^{\mathrm{T}}$ and $\left[\begin{array}{lllll}x_{6} & y_{6} & z_{6}\end{array}\right]^{\mathrm{T}}$ are position vectors of two center points on the axes of joints $R_{5}$ and $R_{6}$. The motion screws in Eq.(2) form a 5-system and a reciprocal constraint screw [39] can be yielded as

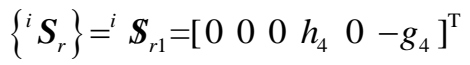

The above constraint screw presents a constraint couple that is perpendicular to the plane formed by two axes of the universal joint. Therefore, with the PMM in phase (a), the reconfigurable hybrid limb has six DOFs and exerts no constraint, while a constraint couple is generated when the PMM evolves into phase (b).

Using two reconfigurable hybrid limbs and a C-limb to connect a moving platform and a fixed base, a three-legged RPM can be constructed as in Fig.5. In limb 1, the axes of joints $R_{11}, R_{12}$ are parallel to the first rotational axis of universal joint $U_{1}$, while the second rotational axis of joint $\mathrm{U}_{1}$ is parallel to the axis of joint $\mathrm{R}_{15}$. The axes of joints $\mathrm{R}_{15}$ and $\mathrm{R}_{26}$ are coincident and perpendicular to the axis of joint $\mathrm{R}_{36}$. The plane of the PMM in limb 2 and line $A_{21} A_{22}$ is perpendicular to the horizontal plane and axis of joint $\mathrm{R}_{11}$. The plane of the PMM in limb 3 is perpendicular to the horizontal plane, while line $A_{31} A_{32}$ is parallel to the axis of joint $\mathrm{R}_{11}$. Five revolute joints, i.e. $\mathrm{R}_{11}, \mathrm{R}_{i 1}$ (revolute joint at point $A_{i 1}, i=2,3$ ) and $\mathrm{R}_{i 2}$ (revolute joint at point $A_{i 2}, i=2,3$ ) are selected as inputs. 


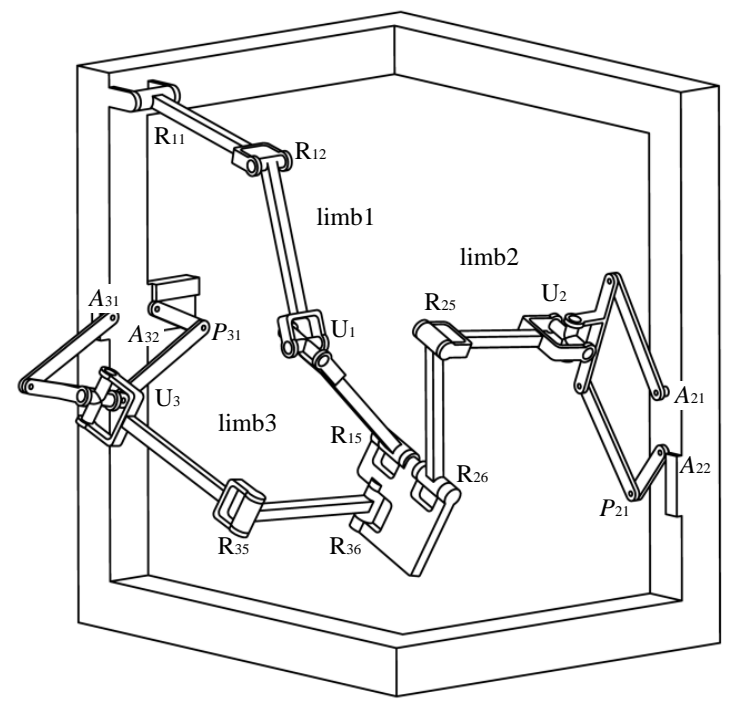

Fig.5 RPM in 3T2R operation mode

The RPM has four configurations with PMMs of the two hybrid limbs in different phases. When the two PMMs are both in phase (a), the RPM locates in a configuration as in Fig.5. In this configuration, limb 1 exerts a constraint couple whose direction is perpendicular to the plane formed by the two axes of universal joint $\mathrm{U}_{1}$. The two hybrid limbs exert no constraint on the moving platform. Therefore, a rotational motion is constrained by the C-limb, the mechanism can work in 3T2R operation mode. All the five input joints are used in this configuration

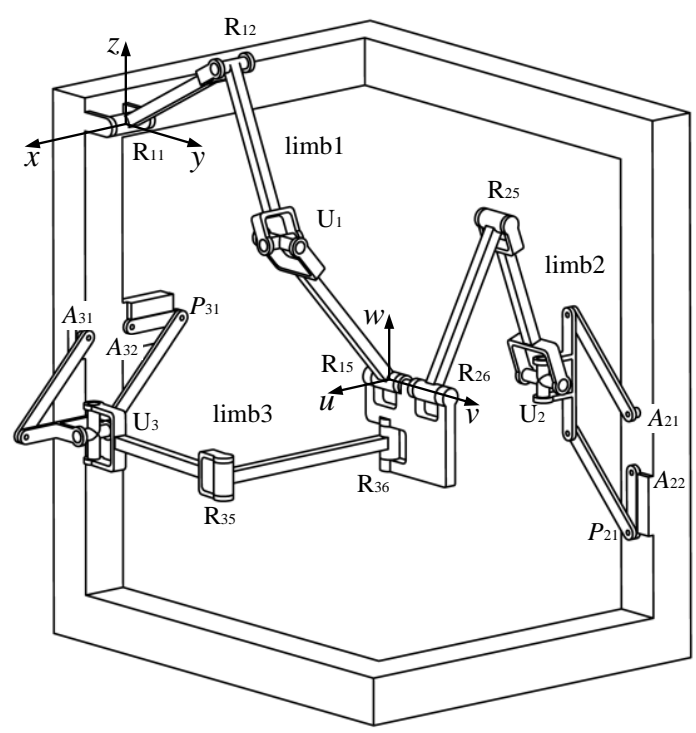

Fig.6 RPM in 3T operation mode

From the 3T2R configuration, if the two PMMs in the hybrid limbs evolve into phase (b) with links $A_{i 2} P_{i 1}(i=2,3)$ annexed with the fixed base, the RPM moves to a configuration as in Fig.6. Attach a global coordinate system $G\{x y z\}$ to the fixed base with its original point located at the center of joint $\mathrm{R}_{11}$. The $x$ axis is aligned with axis of joint $\mathrm{R}_{11}$ and $y$ axis is parallel to the horizontal plane and perpendicular to axis $x$, the $z$ axis is defined according to the right-hand rule. With respect to the global coordinate system, limb 1 exerts a constraint couple in direction $z$, limb 2 exerts a constraint couple in direction $x$, limb 3 exerts a constraint 
couple in direction $y$. These three constraint couples form a wrench system that constraints the rotational motions of the moving platform, the mechanism has three translational motions in this configuration (3T operation mode). Only three input joints are working since the joints at points $A_{22}$ and $A_{32}$ are locked.

When the PMM in limb 2 evolves into phase (b) and that in limb 3 remains in phase (a), only two couples in direction $x$ and $z$ are generated by limb 1 and limb 2. The moving platform has three translational motions and one rotational motion around axis $y$ in such a configuration $\left(3 \mathrm{~T}_{1} \mathrm{R}_{y}\right.$ operation mode). Similarly, the mechanism can work in $3 \mathrm{~T} 1 \mathrm{R}_{x}$ operation mode with the PMM of limb 2 in phase (a) and that of limb 3 in phase (b). These two kinds of 3T1R operation modes both have four working inputs.

Therefore, with the PMMs of the hybrid limbs in different phases, the RPM has the ability to perform 3T motion with 3-DOF, two kinds of 3T1R motion with 4-DOF, 3T2R motion with 5-DOF, which satisfy the DOF requirements in multi-directional AM process. The proposed RPM belongs to the family of metamorphic parallel mechanisms since its reconfigurations are realized through link annexing [40].

\section{Unified kinematic position solutions}

The establishment of unified kinematic models for reconfigurable parallel mechanisms that cover several distinct topologies would be a big challenge $[12,13]$. For the RPM propose here, attach a local coordinate system $L(u v w)$ to the moving platform with its original point located at the center of joint $\mathrm{R}_{15}$. The $u$ axis is perpendicular to the platform plane and $v$ axis is coincident with the axis of joint $\mathrm{R}_{15}$, the $w$ axis is defined according to the right-hand rule, as shown in Fig.6.

The orientation of the moving platform can be expressed by a rotation around axis $x$ followed by a rotation around the resulted $y^{\prime}$ axis. The transformation from the local coordinate system $L(u v w)$ to the global coordinate system $G(x y z)$ can be described by a position vector $\mathbf{P}=[x, y, z]^{T}$, and a rotation matrix ${ }^{G} \mathbf{R}_{L}$, which is expressed as

$$
{ }^{G} \mathbf{R}_{L}=\mathbf{R}_{x} \mathbf{R}_{y}=\left[\begin{array}{ccc}
1 & 0 & 0 \\
0 & \cos \alpha & -\sin \alpha \\
0 & \sin \alpha & \cos \alpha
\end{array}\right]\left[\begin{array}{ccc}
\cos \beta & 0 & \sin \beta \\
0 & 1 & 0 \\
-\sin \beta & 0 & \cos \beta
\end{array}\right]=\left[\begin{array}{ccc}
\cos \beta & 0 & \sin \beta \\
\sin \alpha \sin \beta & \cos \alpha & -\sin \alpha \cos \beta \\
-\cos \alpha \sin \beta & \sin \alpha & \cos \alpha \cos \beta
\end{array}\right]
$$

where $\alpha$ and $\beta$ are rotational angels around axes $x$ and $y^{\prime}$. Let $\theta_{11}, \theta_{i 1}$ and $\theta_{i 2}$ denote the rotational angles of input joints $\mathrm{R}_{11}$, $\mathrm{R}_{i 1}$ and $\mathrm{R}_{i 2}(i=2,3)$, respectively. The inverse and direct kinematic position solutions of the RPM define the position relationships between the rotational angels of input joints and the output parameters $x, y, z, \alpha$ and $\beta$ of the moving platform, and are going to be solved here. Expressing the position vectors of points $A_{11}$ (center point of joint $\mathrm{R}_{11}$ ), $A_{21}$ and $A_{31}$ in global coordinate system $G(x y z)$ as

$$
\mathbf{A}_{11}=[0,0,0]^{T}, \mathbf{A}_{21}=\left[x_{21}, y_{21}, z_{21}\right]^{T}, \mathbf{A}_{31}=\left[x_{31}, y_{31}, z_{31}\right]^{T}
$$


Let $M_{1}, M_{2}$ and $M_{3}$ denote the center points of joint $\mathrm{R}_{15}, \mathrm{R}_{26}$ and $\mathrm{R}_{36}$, respectively. The position vectors of $M_{i}(i=1,2,3)$ expressed in local coordinate system $L(u v w)$ are given as

$$
{ }^{L} \mathbf{M}_{1}=[0,0,0]^{T},{ }^{L} \mathbf{M}_{2}=[0, m, 0]^{T},{ }^{L} \mathbf{M}_{3}=[0,0, n]^{T}
$$

in which the left superscript $L$ denotes that the coordinates are expressed in local system, $|m|$ and $|n|$ are the distances between the center points of $M_{1}$ and $M_{2}, M_{1}$ and $M_{3}$, respectively. The position vectors of $M_{i}(i=1,2,3)$ expressed in global coordinate system $G(x y z)$ can be given by

$$
\mathbf{M}_{i}={ }^{G} \mathbf{R}_{L}{ }^{L} \mathbf{M}_{i}+\mathbf{P}
$$

Substituting Eqs.(4) and (6) into (7) yields

$$
\begin{aligned}
& \mathbf{M}_{1}=[x, y, z]^{T} \\
& \mathbf{M}_{2}=[x, y+m \cos \alpha, z+m \sin \alpha]^{T} \\
& \mathbf{M}_{3}=[x+n \sin \beta, y-n \sin \alpha \cos \beta, z+n \cos \alpha \cos \beta]^{T}
\end{aligned}
$$

The directions of axes $v$ and $w$ with respect to the global coordinate system are given by two unit vectors $\boldsymbol{e}_{1}$ and $\boldsymbol{e}_{2}$ as

$$
\begin{gathered}
\boldsymbol{e}_{1}={ }^{G} \mathbf{R}_{L}[0,1,0]^{T}=[0, \cos \alpha, \sin \alpha]^{T} \\
\boldsymbol{e}_{2}={ }^{G} \mathbf{R}_{L}[0,0,1]^{T}=[\sin \beta,-\sin \alpha \cos \beta, \cos \alpha \cos \beta]^{T}
\end{gathered}
$$

\subsection{Inverse kinematic position solutions}

Inverse position analysis is to find the solutions of $\theta_{11}, \theta_{21}, \theta_{22}, \theta_{31}$ and $\theta_{32}$ with the position and orientation parameters of the moving platform given. The geometrical model of limb 1 is given as in Fig.7, in which $A_{11}, B_{1}, C_{1}$ and $M_{1}$ are used to denote the center points of corresponding joints, $d$ is the norm of vector $\mathbf{A}_{11} \mathbf{C}_{1}, \theta_{1}$ denotes the angel between vector $\mathbf{A}_{11} \mathbf{C}_{1}$ and axis $y$.

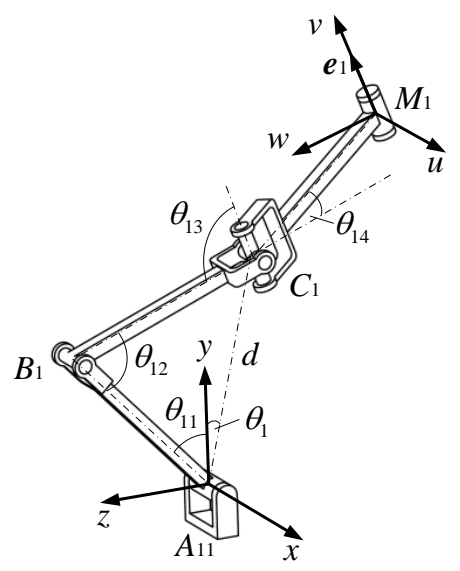

Fig.7 Geometrical model of limb 1

By observing the geometrical relationships in limb 1, a constraint equation is obtained as

$$
\mathbf{C}_{1} \mathbf{M}_{1} \cdot \boldsymbol{e}_{1}=0
$$


The position vector of point $C_{1}$ with respect to the global coordinate system can be given by

$$
\mathbf{C}_{1}=\left[0, d \cos \theta_{1}, d \sin \theta_{1}\right]^{T}
$$

Therefore, vector $\mathbf{C}_{1} \mathbf{M}_{1}$ is obtained as

$$
\mathbf{C}_{1} \mathbf{M}_{1}=\left[x, y-d \cos \theta_{1}, z-d \sin \theta_{1}\right]^{T}
$$

Substituting Eqs. (9) and (13) into (11) yields

$$
\tan \alpha=-\frac{y-d \cos \theta_{1}}{z-d \sin \theta_{1}}
$$

Noticed that the norm of vector $\mathbf{C}_{1} \mathbf{M}_{1}$ is the length of link $C_{1} M_{1}$, which has a constant value $\left(l_{2}\right)$, we have

$$
x^{2}+\left(y-d \cos \theta_{1}\right)^{2}+\left(z-d \sin \theta_{1}\right)^{2}=l_{2}^{2}
$$

The solution of $\theta_{1}$ can be obtained by solving Eqs.(14) and (15) as

$$
\theta_{1}=\arctan \left(\frac{z+\sqrt{\frac{l_{2}^{2}-x^{2}}{1+\tan ^{2} \alpha}}}{y-\tan \alpha \sqrt{\frac{l_{2}^{2}-x^{2}}{1+\tan ^{2} \alpha}}}\right) \text { or } \theta_{1}=\arctan \left(\frac{z-\sqrt{\frac{l_{2}^{2}-x^{2}}{1+\tan ^{2} \alpha}}}{y+\tan \alpha \sqrt{\frac{l_{2}^{2}-x^{2}}{1+\tan ^{2} \alpha}}}\right)
$$

The initial assembly mode of limb 1 would suggest how to chose the actual solution for $\theta_{1}$ from the two in Eq.(16). Then, value of $d$ can be obtained by substituting $\theta_{1}$ into Eq.(14). Provided that the length of link $A_{11} B_{1}$ and $B_{1} C_{1}$ are both $l_{1}$, a triangle equation is established as

$$
\cos \left(\theta_{11}-\theta_{1}\right)=\frac{d}{2 l_{1}}
$$

The solution of $\theta_{11}$ can thus be obtained.

Fig.8 shows the geometrical model of limb 2, where $C_{2}$ denotes the center point of universal joint $\mathrm{U}_{2}, \boldsymbol{s}_{2}$ is a unit vector that represents the direction of the first rotational axis of $\mathrm{U}_{2}$.

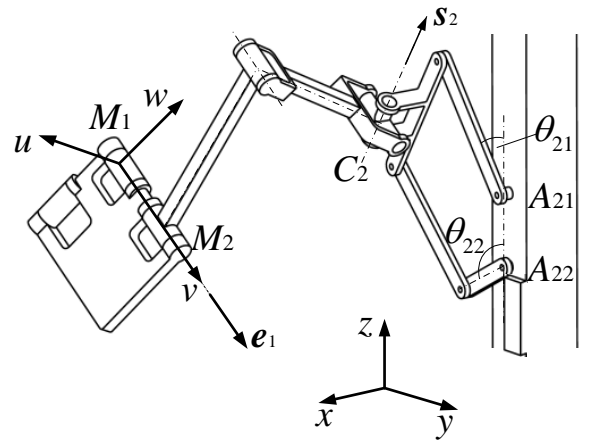

Fig.8 Geometrical model of limb 2

Vector $s_{2}$ and the position vector of point $C_{2}$ expressed in global coordinate system can be given by 


$$
\begin{gathered}
\boldsymbol{s}_{2}=\left[0,-\sin \gamma_{2}, \cos \gamma_{2}\right]^{T} \\
\mathbf{C}_{2}=\left[x_{21}, y_{21}+l \sin \gamma_{2} / 2-l \sin \theta_{21}, z_{21}+l \cos \theta_{21}-l \cos \gamma_{2} / 2\right]^{T}
\end{gathered}
$$

where $\gamma_{2}$ is the angel between $\boldsymbol{s}_{2}$ and axis $z$. Vector $\mathbf{C}_{2} \mathbf{M}_{2}$ is obtained as

$$
\mathbf{C}_{2} \mathbf{M}_{2}=\left[x-x_{21}, y+m \cos \alpha-y_{21}-l \sin \gamma_{2} / 2+l \sin \theta_{21}, z+m \sin \alpha-z_{21}-l \cos \theta_{21}+l \cos \gamma_{2} / 2\right]^{T}
$$

It is easy to find that

$$
\begin{gathered}
\boldsymbol{e}_{1} \cdot \boldsymbol{s}_{2}=0 \\
\boldsymbol{e}_{1} \cdot \mathbf{C}_{2} \mathbf{M}_{2}=0
\end{gathered}
$$

Substituting Eqs. (9) and (18) into (21) yields

$$
\gamma_{2}=\alpha
$$

Substituting Eqs. (9) and (20) into (22) yields

$$
\tan \alpha=-\frac{y+m \cos \alpha-y_{21}-l \sin \gamma_{2} / 2+l \sin \theta_{21}}{z+m \sin \alpha-z_{21}-l \cos \theta_{21}+l \cos \gamma_{2} / 2}
$$

The solution of $\theta_{21}$ can be obtained through Eq.(24). A closed loop equation for the PMM in limb 2 is established as

$$
\left(l \cos \theta_{21}-l \cos \gamma_{2}-l \cos \theta_{22} / 2+l / 2\right)^{2}+\left(l \sin \theta_{21}-l \sin \gamma_{2}-l \sin \theta_{22} / 2\right)^{2}=l^{2}
$$

Through which the solution of $\theta_{22}$ can be obtained.

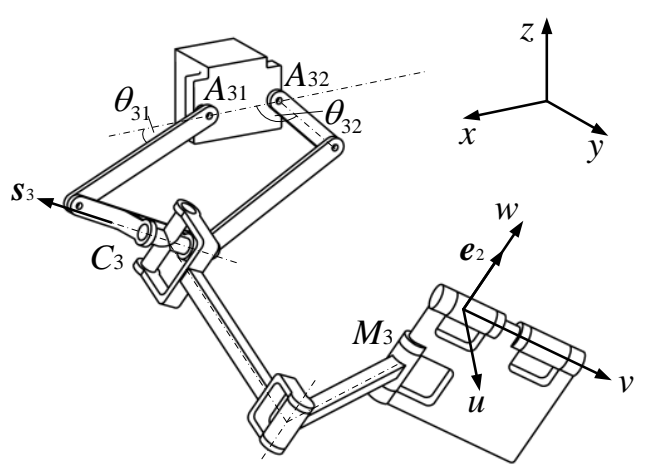

Fig.9 Geometrical model of limb 3

The geometrical model for kinematic position analysis of limb 3 is given as in Fig.9, where $C_{3}$ denotes the center point of universal joint $\mathrm{U}_{3}, s_{3}$ is a unit vector that represents the direction of the first rotational axis of $\mathrm{U}_{3}$.

Vector $s_{3}$ and the position vector of point $C_{3}$ expressed in global coordinate system can be given by

$$
\begin{gathered}
\boldsymbol{s}_{3}=\left[\cos \gamma_{3}, 0,-\sin \gamma_{3}\right]^{T} \\
\mathbf{C}_{3}=\left[x_{31}+l \cos \theta_{31}-l \cos \gamma_{3} / 2, y_{31}, z_{31}-l \sin \theta_{31}+l \sin \gamma_{3} / 2\right]^{T}
\end{gathered}
$$

where $\gamma_{3}$ is the angel between $\boldsymbol{s}_{3}$ and axis $x$. Vector $\mathbf{C}_{3} \mathbf{M}_{3}$ is obtained as 


$$
\mathbf{C}_{3} \mathbf{M}_{3}=\left[\begin{array}{c}
x+n \sin \beta-x_{31}-l \cos \theta_{31}+l \cos \gamma_{3} / 2 \\
y-n \sin \alpha \cos \beta-y_{31} \\
z+n \cos \alpha \cos \beta-z_{31}+l \sin \theta_{31}-l \sin \gamma_{3} / 2
\end{array}\right]
$$

It is easy to find that

$$
\begin{gathered}
\boldsymbol{e}_{2} \boldsymbol{s}_{3}=0 \\
\boldsymbol{e}_{2} \cdot \mathbf{C}_{3} \mathbf{M}_{3}=0
\end{gathered}
$$

Substituting Eqs.(10) and (26) into (29) yields

$$
\gamma_{3}=\arctan \left(\frac{\tan \beta}{\cos \alpha}\right)
$$

Substituting Eqs. (10) and (28) into (30) yields

$$
\begin{aligned}
& \sin \beta\left(x+n \sin \beta-x_{31}-l \cos \theta_{31}+l \cos \gamma_{3} / 2\right) \\
& -\sin \alpha \cos \beta\left(y-n \sin \alpha \cos \beta-y_{31}\right) \\
& +\cos \alpha \cos \beta\left(z+n \cos \alpha \cos \beta-z_{31}+l \sin \theta_{31}-l \sin \gamma_{3} / 2\right)=0
\end{aligned}
$$

The solution of $\theta_{31}$ can be obtained through Eq.(32). A closed loop equation for the PMM in limb 3 is established as

$$
\left(l \cos \theta_{31}-l \cos \gamma_{3}-l \cos \theta_{32} / 2+l / 2\right)^{2}+\left(l \sin \theta_{31}-l \sin \gamma_{3}-l \sin \theta_{32} / 2\right)^{2}=l^{2}
$$

Through which $\theta_{32}$ can be solved.

\subsection{Forward kinematic position solutions}

For the forward position analysis, input angels $\theta_{11}, \theta_{21}, \theta_{22}, \theta_{31}$ and $\theta_{32}$ are given, output parameters $x, y, z, \alpha$ and $\beta$ are desired to be determined, the solution procedure is given here.

Firstly, $\gamma_{2}$ and $\gamma_{3}$ can be obtained through Eqs.(25) and (33), respectively. The orientation parameters $\alpha$ and $\beta$ of the moving platform are thus derived through Eqs.(23) and (31) as

$$
\begin{gathered}
\alpha=\gamma_{2} \\
\beta=\arctan \left(\cos \gamma_{2} \tan \gamma_{3}\right)
\end{gathered}
$$

Noticed that $A_{11} B_{1}=B_{1} C_{1}=l_{1}$, we have

$$
\left(d \cos \theta_{1}-l_{1} \cos \theta_{11}\right)^{2}+\left(d \sin \theta_{1}-l_{1} \sin \theta_{11}\right)^{2}=l_{1}^{2}
$$

Combining Eq.(14) and Eq.(36), we can represent $d \cos \theta_{1}$ and $d \sin \theta_{1}$ with expressions that contain variables $y$ and $z$, Substituting the results into Eq.(15), an equation contains unknown variables $x, y, z$ can be obtained, which can be written in general form as

$$
f\left(x^{2}, y^{2}, z^{2}\right)=0
$$

Eqs.(24) and (32) can be written in general forms as 


$$
\begin{gathered}
f(y, z)=0 \\
f(x, y, z)=0
\end{gathered}
$$

With the three independent Eqs.(37-39), position parameters $x, y$ and $z$ can be solved easily.

A parallel mechanism usually has more than one set of direct position solution. Through Eqs.(25) and (33), it is easy to find that $\gamma_{i}(i=2,3)$ will have two solutions(one positive and one negative) with a set of $\theta_{i 1}$ and $\theta_{i 2}$ given. Therefore, both $\alpha$ and $\beta$ have two possible solutions. For the position parameters, four sets of $x, y$ and $z$ are expected to be obtained since Eqs.(36) and (37) are quadratic equations. Therefore, the RPM has as many as sixteen sets of direct position solutions with one set of inputs in $3 \mathrm{~T} 2 \mathrm{R}$ operation mode. It should be noted that in 3T1R and 3T operation modes, $\theta_{22}$ and/or $\theta_{32}$ has a constant value $180^{\circ}, \alpha$ and/or $\beta$ will be zero, the number of direct position solution sets will decrease. Eight and four sets of direct position solutions will be obtained with the mechanism in $3 \mathrm{~T} 1 \mathrm{R}$ and $3 \mathrm{~T}$ operation modes, respectively.

\subsection{Numerical and simulation results}

According to the above procedure, the direct kinematic position solutions can be easily obtained with the help of math software. In this section, numerical results obtained through MATHEMATICAS are compared with simulation results in ADAMS. The architectural parameters of the RPM are given in Table.1.

Table 1. Architectural parameters of the RPM

\begin{tabular}{ll}
\hline \hline Parameter & Value $(\mathrm{mm})$ \\
\hline $\mathrm{m}$ & 120.2 \\
$\mathrm{n}$ & -100.1 \\
$l$ & 200 \\
$l_{1}$ & 250 \\
$l_{2}$ & 300 \\
${ }^{\mathrm{G}} \mathbf{A}_{21}$ & {$[-338,436,-442]^{\mathrm{T}}$} \\
${ }^{\mathrm{G}} \mathbf{A}_{31}$ & {$[-31,-21,-362]^{\mathrm{T}}$} \\
\hline \hline
\end{tabular}

Since the RPM has four operation modes, we give four sets of inputs accordingly. The initial position is defined as $\theta_{11}=40^{\circ}$, $\theta_{21}=25^{\circ}, \theta_{22}=180^{\circ}, \theta_{31}=25^{\circ}$ and $\theta_{32}=180^{\circ}$. The four sets of inputs are as follows

Input 1: $\theta_{11}=40-5 t, \theta_{21}=25+5 t, \theta_{22}=180, \theta_{31}=25+5 t, \theta_{32}=180, t \in[0 \square 9 s]$

Input 2: $\theta_{11}=40-5 t, \theta_{21}=25+5 t, \theta_{22}=180-5 t, \theta_{31}=25+5 t, \quad \theta_{32}=180, t \in[0 \square 9 s]$

Input 3: $\theta_{11}=40-5 t, \theta_{21}=25+5 t, \theta_{22}=180, \theta_{31}=25+5 t, \theta_{32}=180-5 t, \quad t \in[0 \square 9 s]$

Input 4: $\theta_{11}=40-5 t, \theta_{21}=25+5 t, \theta_{22}=180-5 t, \quad \theta_{31}=25+5 t, \quad \theta_{32}=180-10 t, \quad t \in[0 \square 9 s]$

in which, input 1 corresponds to $3 \mathrm{~T}$ operation mode, input 2 corresponds to $3 \mathrm{~T}_{1} \mathrm{R}_{x}$ operation mode, input 3 corresponds to $3 \mathrm{~T} 1 \mathrm{R}_{y}$ operation mode and input 4 corresponds to 3T2R operation mode. For simplify, we calculate the output parameters $x, y, z, \alpha$ and $\beta$ at time $0 s, 1 s, 2 s, 3 s, 4 s, 5 s, 6 s, 7 s, 8 s, 9 s$ for all the four sets of inputs, chose the reasonable result values according to the 
initial assembly mode of the mechanism and then plot interpolation curves with those values, resulting in curves of output parameters $x, y, z, \alpha$ and $\beta$ as in Fig.10(a), Fig.11(a), Fig.12(a), Fig.13(a) and Fig.14(a), respectively (The values of $\alpha$ with input 1 , input 3 and $\beta$ with input 1 , input 2 are zero, so those curves are not plotted).

Simulations in ADAMS associated with the four sets of inputs are performed and the results are plotted in Fig. 10(b), Fig.11(b), Fig.12(b), Fig.13(b) and Fig.14(b). It can be observed that the numerical results well match the simulation results, which demonstrate that the direct position solution procedures are reliable.

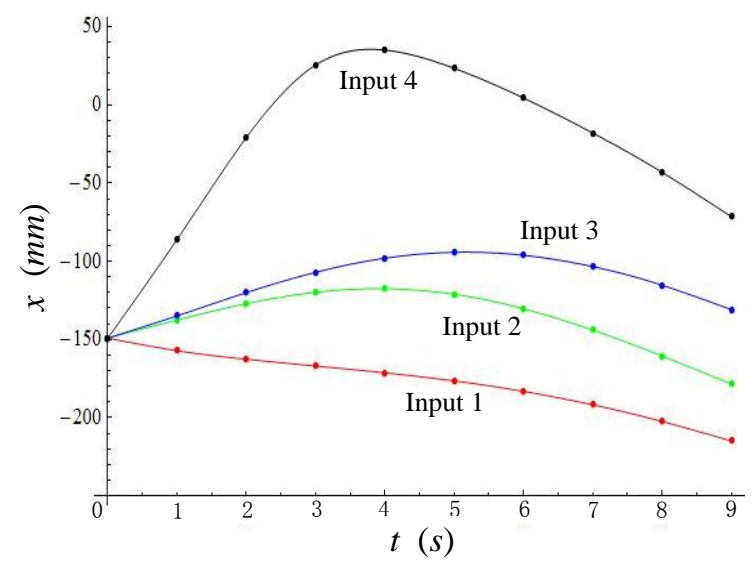

(a)

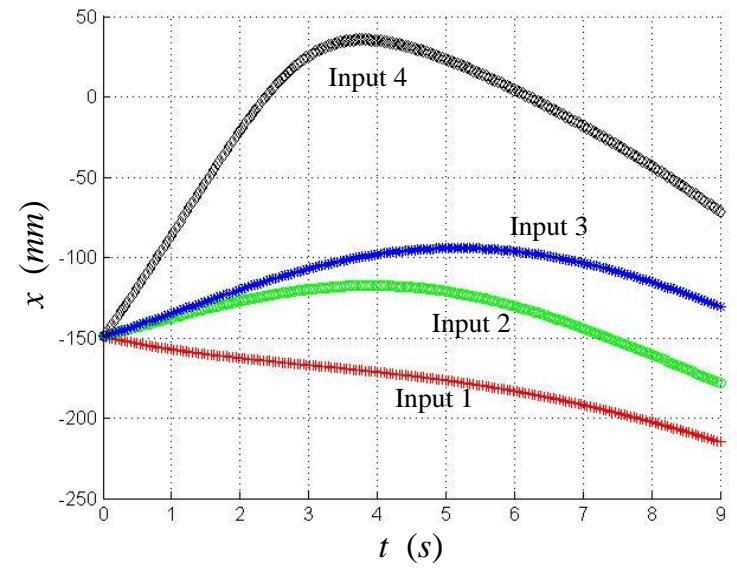

(b)

Fig.10 Curves of $x$ for four sets of inputs: (a) numerical results (b) simulation results

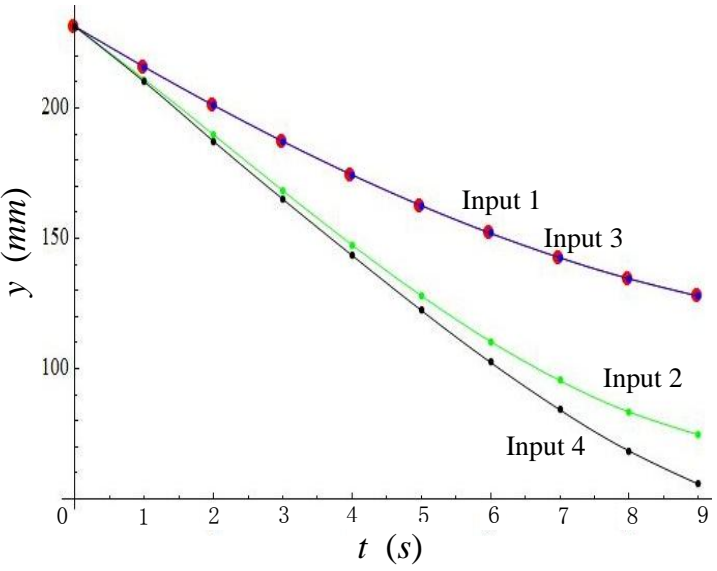

(a)

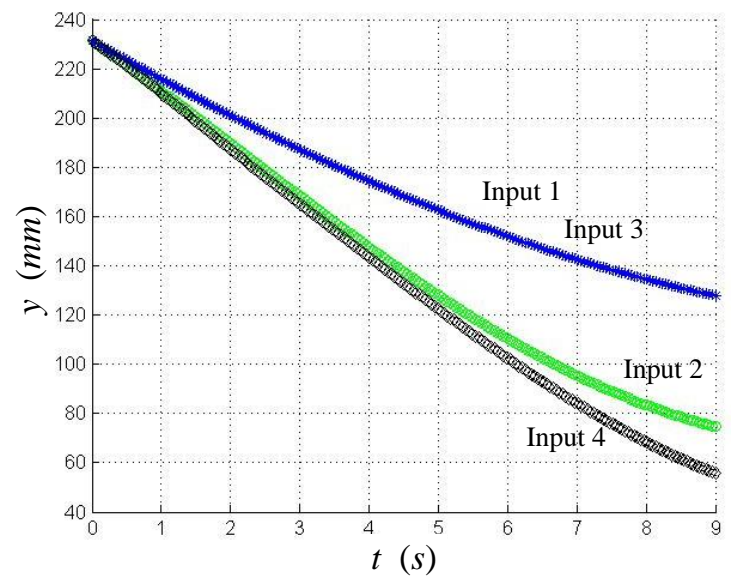

(b)

Fig.11 Curves of $y$ for four sets of inputs: (a) numerical results (b) simulation results 


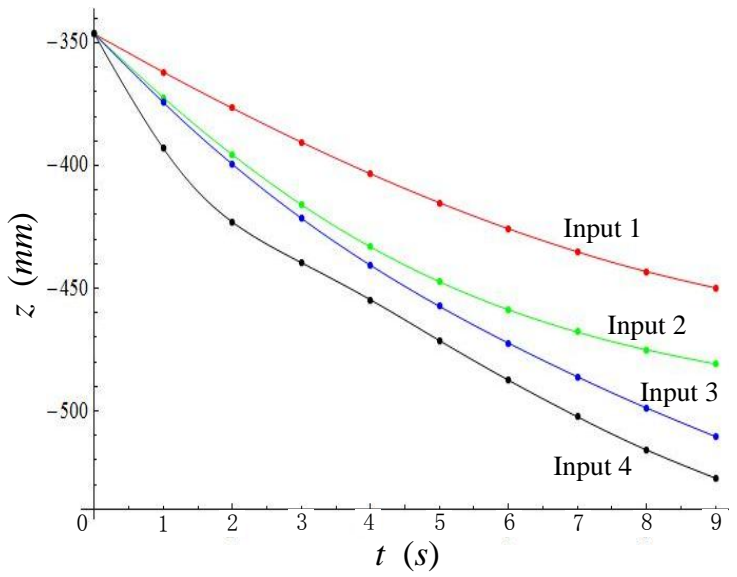

(a)

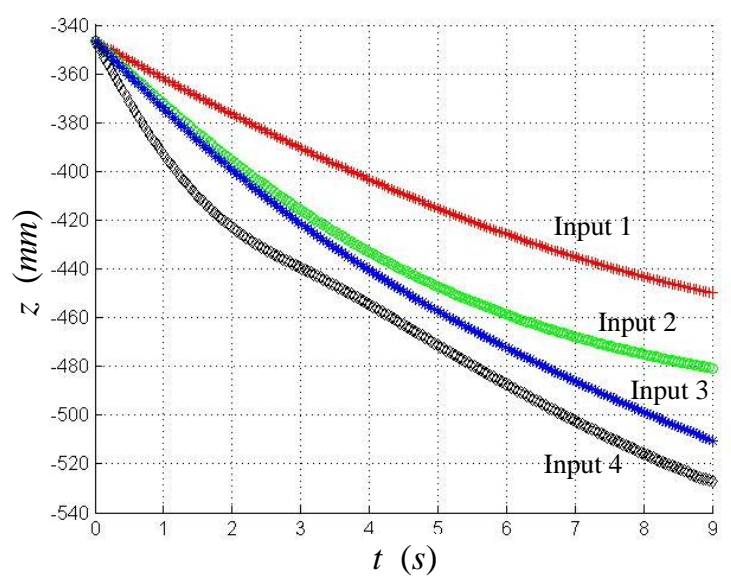

(b)

Fig.12 Curves of $\mathrm{z}$ for four sets of inputs: (a) numerical results (b) simulation results

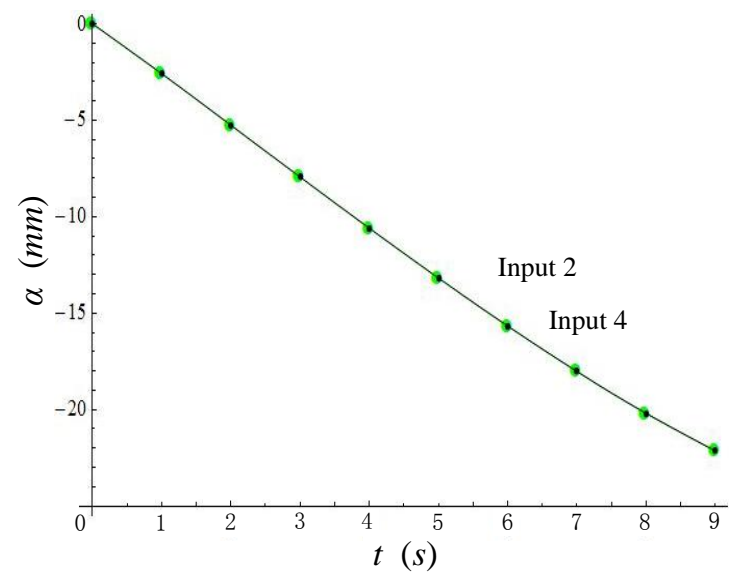

(a)

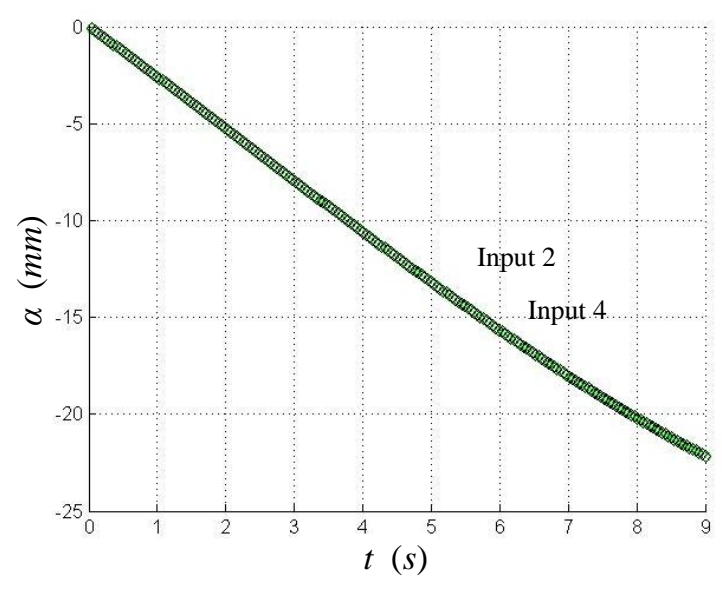

(b)

Fig.13 Curves of $\alpha$ for four sets of inputs: (a) numerical results (b) simulation results

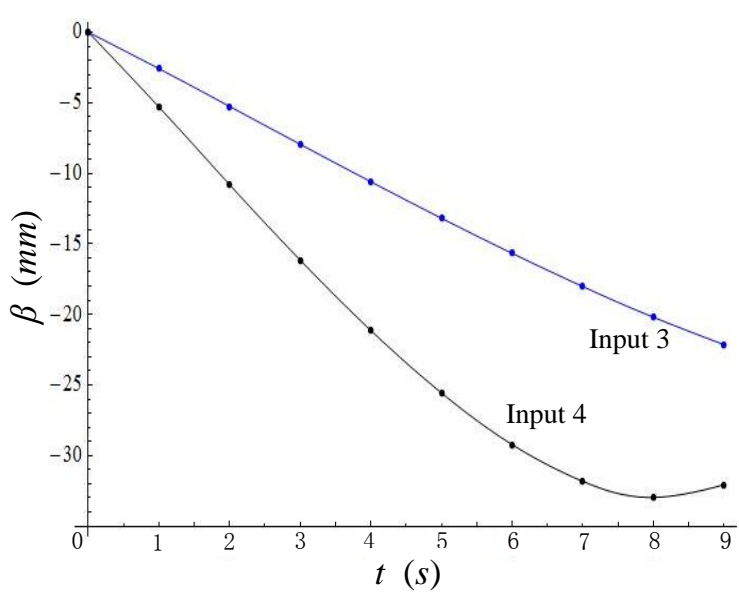

(a)

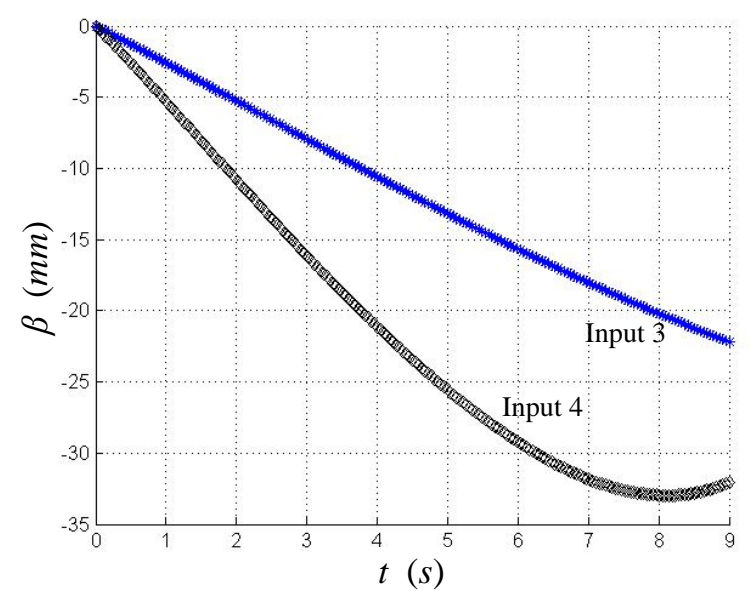

(b)

Fig.14 Curves of $\beta$ for four sets of inputs: (a) numerical results (b) simulation results 


\section{Jacobian matrix}

Representing the relationship between the input joint rates and the moving platform output velocity, Jacobian matrix plays an important role in performance evaluation of parallel mechanisms. For the RPM proposed here, a unified Jacobian model that covers all the configurations is desired. The method based on reciprocal screw [21, 41] that is efficient in establishment of Jacobian matrix and will provide convenience in comprehensive description of singularity types will be used here.

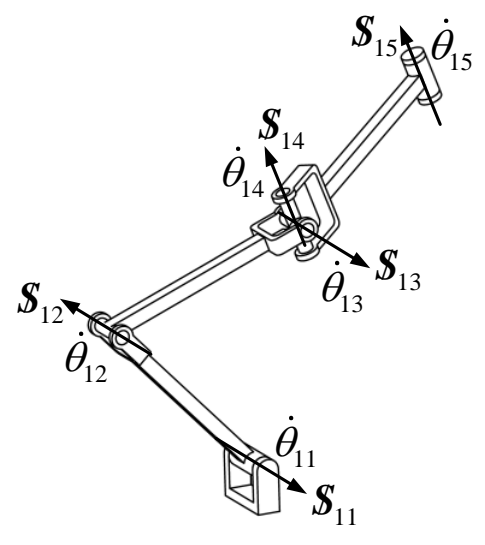

Fig.15 Twists in limb 1

Let $\boldsymbol{S}_{p}=\left[\begin{array}{ll}\boldsymbol{w}^{\mathrm{T}} & \boldsymbol{v}_{p}^{\mathrm{T}}\end{array}\right]^{\mathrm{T}}$ be the instantaneous twist of the moving platform expressed in the global coordinate system, where the primal part, $\boldsymbol{w}$ represents the angular velocity of the moving platform and the dual part, $\boldsymbol{v}_{p}$, is the linear velocity of a point in the moving platform that is instantaneously coincident with the origin of the global coordinate system. $\boldsymbol{S}_{p}$ can be expressed as a linear combination of five twists associated with joints in limb 1 as

$$
\boldsymbol{S}_{p}=\dot{\theta}_{11} \boldsymbol{S}_{11}+\dot{\theta}_{12} \boldsymbol{S}_{12}+\dot{\theta}_{13} \boldsymbol{S}_{13}+\dot{\theta}_{14} \boldsymbol{S}_{14}+\dot{\theta}_{15} \boldsymbol{S}_{15}
$$

where $\dot{\theta}_{1 i}$ denotes the intensity and $\boldsymbol{S}_{1 i}$ is a unit screw corresponding to the $i$ th joint in limb $1(i=1,2,3,4,5)$, as in Fig.15. A constraint screw $\boldsymbol{S}_{r 1 C}$ that is reciprocal to all the joint screws can be identified with the following equation satisfied

$$
\boldsymbol{S}_{r 1 C}^{T} \boldsymbol{S}_{p}=0
$$

On the other hand, a screw $\boldsymbol{S}_{r 11}$ that is reciprocal to all the joint screws, except for the actuated joint screw $\boldsymbol{S}_{11}$ can be found. The physical meaning of this screw is a force exerted by the actuated joint. Taking the orthogonal product of both sides of Eq.(40) with $\boldsymbol{S}_{r 11}$ yields

$$
\boldsymbol{S}_{r 11}^{T} \boldsymbol{S}_{p}=\boldsymbol{S}_{r 11}^{T} \boldsymbol{S}_{11} \dot{\theta}_{11}
$$

In limb $i(i=2,3)$, the motion of link $P_{i 2} P_{i 3}$ in the PMM is constrained only by the chain consisting of joints at points $A_{i 1}$ and $P_{i 3}$, the equivalent twist of link $P_{i 2} P_{i 3}$ can be expressed with twists associated with those two joints. Therefore, $\boldsymbol{S}_{p}$ can be written as a linear combination of twists as 


$$
\boldsymbol{S}_{p}=\dot{\theta}_{i 1} \boldsymbol{S}_{i 1}+\dot{\theta}_{i e} \boldsymbol{S}_{i e}+\dot{\theta}_{i 3} \boldsymbol{S}_{i 3}+\dot{\theta}_{i 4} \boldsymbol{S}_{i 4}+\dot{\theta}_{i 5} \boldsymbol{S}_{i 5}+\dot{\theta}_{i 6} \boldsymbol{S}_{i 6} \quad(i=2,3)
$$

in which $\boldsymbol{S}_{i 1}$ is the twist of the actuated joint at point $A_{i 1}, \boldsymbol{S}_{i e}$ is the twist of joint at point $P_{i 3}, \boldsymbol{S}_{i 3}, \boldsymbol{S}_{i 4}, \boldsymbol{S}_{i 5}$ and $\boldsymbol{S}_{i 6}$ are twists of the universal joint and revolute joints, as in Fig.16.

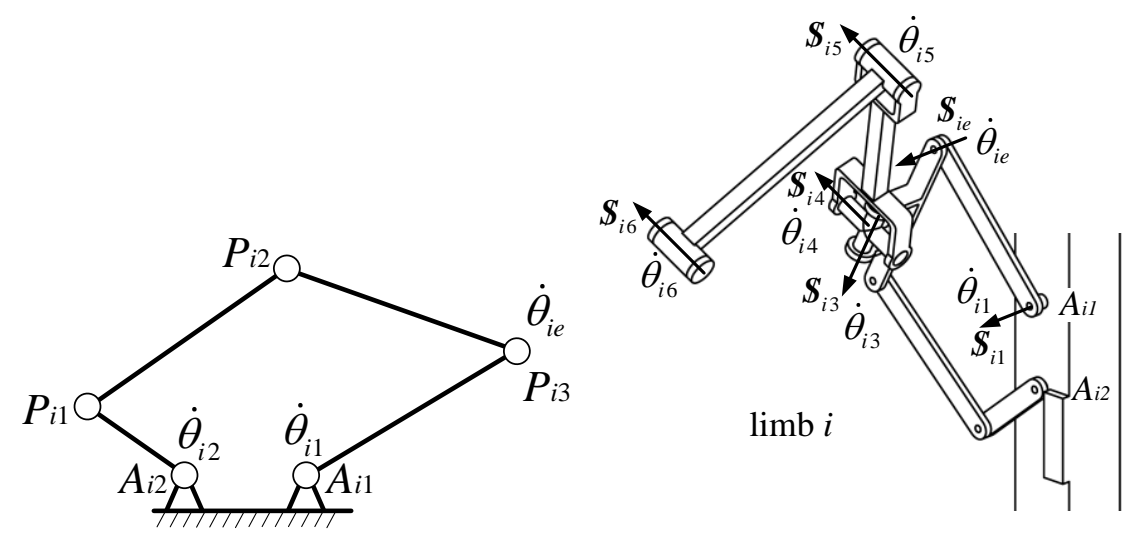

Fig.16 Twists in limb $i(i=2,3)$

A screw $\boldsymbol{S}_{r i 1}$ that is reciprocal to all the joint screws, except for the actuated joint screw $\boldsymbol{S}_{i 1}$ can be found, which is a force wrench that passes the axis of $\boldsymbol{S}_{i e}$ and $\boldsymbol{S}_{i 3}$, and parallel to the axes of $\boldsymbol{S}_{i 4}, \boldsymbol{S}_{i 5}$ and $\boldsymbol{S}_{i 6}$. Taking the orthogonal product of both sides of Eq.(43) with $\boldsymbol{S}_{r i 1}$ yields

$$
\boldsymbol{S}_{r i 1}^{T} \boldsymbol{S}_{p}=\boldsymbol{S}_{r i 1}^{T} \boldsymbol{S}_{i 1} \dot{\theta}_{i 1}
$$

Meanwhile, the other screw $\boldsymbol{S}_{r i e}$ that is reciprocal to all the joint screws, except for joint screw $\boldsymbol{S}_{i e}$ can be found, which is a force wrench that passes the axis of $\boldsymbol{S}_{i 1}$ and $\boldsymbol{S}_{i 3}$, and parallel to the axes of $\boldsymbol{S}_{i 4}, \boldsymbol{S}_{i 5}$ and $\boldsymbol{S}_{i 6}$. Taking the orthogonal product of both sides of Eq.(43) with $\boldsymbol{S}_{\text {rie }}$ yields

$$
\boldsymbol{S}_{r i e}^{T} \boldsymbol{S}_{p}=\boldsymbol{S}_{r i e}^{T} \boldsymbol{S}_{i e} \dot{\theta}_{i e}
$$

The PMM in Fig.16 can be regarded as a mechanism with parallel structure, therefore, the angular velocity $\dot{\theta}_{i e}$ can be written as the combination of input joint rates as

$$
\dot{\theta}_{i e}=a_{i 1} \dot{\theta}_{i 1}+a_{i 2} \dot{\theta}_{i 2}
$$

where $a_{i 1}$ and $a_{i 2}$ are coefficients that are dependent with the dimension and configuration of the PMM. Substituting Eq (46) into Eq (45) yields

$$
\boldsymbol{S}_{r i e}^{T} \boldsymbol{S}_{p}=a_{i 1} \boldsymbol{S}_{r i e}^{T} \boldsymbol{S}_{i e} \dot{\theta}_{i 1}+a_{i 2} \boldsymbol{S}_{r i e}^{T} \boldsymbol{S}_{i e} \dot{\theta}_{i 2}
$$

Since $\boldsymbol{S}_{r i 1}$ is parallel to $\boldsymbol{S}_{\text {rie }}, \boldsymbol{S}_{r i 1}$ intersects with $\boldsymbol{S}_{i e}, \boldsymbol{S}_{\text {rie }}$ intersects with $\boldsymbol{S}_{i 1}$, we can easily calculate that $\boldsymbol{S}_{r i 1}^{T} \boldsymbol{S}_{i 1}=-\boldsymbol{S}_{r i e}^{T} \boldsymbol{S}_{i e}$. A matrix-form expression is obtained as 


$$
\left[\begin{array}{c}
\boldsymbol{S}_{r i 1}^{T} \\
\boldsymbol{S}_{r i e}^{T}
\end{array}\right] \boldsymbol{S}_{p}=\left[\begin{array}{cccccc}
0 & \boldsymbol{S}_{r i 1}^{T} \boldsymbol{S}_{i 1} & 0 & 0 & 0 & 0 \\
0 & -a_{i 1} \boldsymbol{S}_{r i 1}^{T} \boldsymbol{S}_{i 1} & -a_{i 2} \boldsymbol{S}_{r i 1}^{T} \boldsymbol{S}_{i 1} & 0 & 0 & 0
\end{array}\right]\left[\begin{array}{c}
\dot{\theta}_{i 1} \\
\dot{\theta}_{i 2}
\end{array}\right]
$$

It should noted that when the PMM is in phase (b), $a_{i 1}=-1, a_{i 2}=0, \dot{\theta}_{i 2}=0$, Eq.(48) can be reduced as

$$
\left[\begin{array}{l}
\boldsymbol{S}_{r i 1}^{T} \\
\boldsymbol{S}_{r i c}^{T}
\end{array}\right] \boldsymbol{S}_{p}=\left[\begin{array}{cccccc}
0 & \boldsymbol{S}_{r i 1}^{T} \boldsymbol{S}_{i 1} & 0 & 0 & 0 & 0 \\
0 & 0 & 0 & 0 & 0 & 0
\end{array}\right]\left[\begin{array}{c}
\dot{\theta}_{i 1} \\
0
\end{array}\right]
$$

where $\boldsymbol{S}_{r i c}$ is a wrench whose primal part is $\mathbf{0}$. In this condition, $\boldsymbol{S}_{r i c}$ is a constraint couple that constrains a rotational DOF of the moving platform.

Casting Eqs. $(41,42,48)$ in matrix -vector form and simplifying the result as

$$
\left[\begin{array}{l}
\boldsymbol{S}_{r 11}^{T} \\
\boldsymbol{S}_{r 21}^{T} \\
\boldsymbol{S}_{r 2 e}^{T} \\
\boldsymbol{S}_{r 31}^{T} \\
\boldsymbol{S}_{r 3 e}^{T} \\
\boldsymbol{S}_{r 1 C}^{T}
\end{array}\right] \boldsymbol{S}_{p}^{T}=\left[\begin{array}{cccccc}
\boldsymbol{S}_{r 11}^{T} \boldsymbol{S}_{11} & 0 & 0 & 0 & 0 & 0 \\
0 & \boldsymbol{S}_{r 21}^{T} \boldsymbol{S}_{21} & 0 & 0 & 0 & 0 \\
0 & -a_{21} \boldsymbol{S}_{r 21}^{T} \boldsymbol{S}_{21} & -a_{22} \boldsymbol{S}_{r 21}^{T} \boldsymbol{S}_{21} & 0 & 0 & 0 \\
0 & 0 & 0 & \boldsymbol{S}_{r 31}^{T} \boldsymbol{S}_{31} & 0 & 0 \\
0 & 0 & 0 & -a_{31} \boldsymbol{S}_{r 31}^{T} \boldsymbol{S}_{31} & -a_{32} \boldsymbol{S}_{r 31}^{T} \boldsymbol{S}_{31} & 0 \\
0 & 0 & 0 & 0 & 0 & 0
\end{array}\right]\left[\begin{array}{c}
\dot{\theta}_{11} \\
\dot{\theta}_{21} \\
\dot{\theta}_{22} \\
\dot{\theta}_{31} \\
\dot{\theta}_{32} \\
0
\end{array}\right]
$$

The relationship between the input joint rates and the moving platform output velocity is thus established, which can be written in general form as

$$
\boldsymbol{J}_{x} \dot{\boldsymbol{x}}=\boldsymbol{J}_{q} \dot{\boldsymbol{q}}
$$

where

$$
\boldsymbol{J}_{x}=\left[\begin{array}{c}
\boldsymbol{S}_{r 11}^{T} \\
\boldsymbol{S}_{r 21}^{T} \\
\boldsymbol{S}_{r 2 e}^{T} \\
\boldsymbol{S}_{r 31}^{T} \\
\boldsymbol{S}_{r 3 e}^{T} \\
\boldsymbol{S}_{r 1 C}^{T}
\end{array}\right], \quad \boldsymbol{J}_{q}^{T}=\left[\begin{array}{cccccc}
\boldsymbol{S}_{r 11}^{T} \boldsymbol{S}_{11} & 0 & 0 & 0 & 0 & 0 \\
0 & \boldsymbol{S}_{r 21}^{T} \boldsymbol{S}_{21} & 0 & 0 & 0 & 0 \\
0 & 0 & a_{21} \boldsymbol{S}_{r 2 e}^{T} \boldsymbol{S}_{2 e} & a_{22} \boldsymbol{S}_{r 2 e}^{T} \boldsymbol{S}_{2 e} & 0 & 0 \\
0 & 0 & 0 & \boldsymbol{S}_{r 31}^{T} \boldsymbol{S}_{31} & 0 & 0 \\
0 & 0 & 0 & 0 & a_{31} \boldsymbol{S}_{r 3 e}^{T} \boldsymbol{S}_{3 e} & a_{32} \boldsymbol{S}_{r 3 e}^{T} \boldsymbol{S}_{3 e} \\
0 & 0 & 0 & 0 & 0 & 0
\end{array}\right]
$$

The overall Jacobian matrix, $\boldsymbol{J}$, can be written as

$$
\boldsymbol{J}=\boldsymbol{J}_{q}^{-1} \boldsymbol{J}_{x}
$$

The aforementioned analysis builds a unified Jacobian model for the RPM. Relationship between the input joint rates and the moving platform output velocity in all the operation modes can be derived through this model. For example, when the RPM is in 3T operation mode, $a_{21}=a_{31}=-1, a_{22}=a_{32}=0, \dot{\theta}_{22}=\dot{\theta}_{32}=0$, Eq. (50) can be reduced as 


$$
\left[\begin{array}{c}
\boldsymbol{S}_{r 11}^{T} \\
\boldsymbol{S}_{r 21}^{T} \\
\boldsymbol{S}_{r 2 C}^{T} \\
\boldsymbol{S}_{r 31}^{T} \\
\boldsymbol{S}_{r 3 C}^{T} \\
\boldsymbol{S}_{r 1 C}^{T}
\end{array}\right] \boldsymbol{S}_{p}=\left[\begin{array}{cccccc}
\boldsymbol{S}_{r 11}^{T} \boldsymbol{S}_{11} & 0 & 0 & 0 & 0 & 0 \\
0 & \boldsymbol{S}_{r 21}^{T} \boldsymbol{S}_{21} & 0 & 0 & 0 & 0 \\
0 & 0 & 0 & 0 & 0 & 0 \\
0 & 0 & 0 & \boldsymbol{S}_{r 31}^{T} \boldsymbol{S}_{31} & 0 & 0 \\
0 & 0 & 0 & 0 & 0 & 0 \\
0 & 0 & 0 & 0 & 0 & 0
\end{array}\right]\left[\begin{array}{c}
\dot{\theta}_{11} \\
\dot{\theta}_{21} \\
0 \\
\dot{\theta}_{31} \\
0 \\
0
\end{array}\right]
$$

In this condition, $\boldsymbol{S}_{r 11}, \boldsymbol{S}_{r 21}$ and $\boldsymbol{S}_{r 31}$ are actuation force wrenches exerted by the three working inputs, while $\boldsymbol{S}_{r 1 C}$, $\boldsymbol{S}_{r 2 C}$ and $\boldsymbol{S}_{r 3 C}$ are couple wrenches that constraint the rotational DOFs of the moving platform. Similarly, the detailed Jacobian matrices in two 3T1R operation modes can also be obtained with the unified model.

\section{Singularity analysis}

Generally, singularities of a parallel mechanism can be classified into three types [42]. The first type is inverse kinematic singularity, also called limb singularity [37]. This kind of singularities usually occurs at the workspace boundary. The mechanism loses one or more degrees of freedom at these configurations. The second type is direct kinematic singularity that includes platform singularity and actuation singularity. A mechanism is out of control at direct kinematic singularities. The third type is combined singularity, which occurs when an inverse kinematic singularity and a direct kinematic singularity take place at the same time. This kind of singularities can be easily identified as long as the inverse kinematic singularities and direct kinematic singularities are determined, and thus be ignored here.

\subsection{Inverse kinematic singularity}

Inverse kinematic singularities will occur when the twist system of a limb is degenerated. Since the three limbs in the RPM have different structures, their singularity conditions will be discussed respectively.

\subsubsection{Inverse kinematic singularity of limb 1}

The five twists of limb 1 given in Eq.(40) form a twist system $\boldsymbol{S}_{1}=\left[\begin{array}{lllll}\boldsymbol{S}_{11}^{T} & \boldsymbol{S}_{12}^{T} & \boldsymbol{S}_{13}^{T} & \boldsymbol{S}_{14}^{T} & \boldsymbol{S}_{15}^{T}\end{array}\right]^{T}$. Inverse kinematic singularities occur in two conditions with the twist system $\boldsymbol{S}_{1}$ degenerated.

Condition 1: when limb 1 moves to the configuration with axes of joints $R_{11}, R_{12}$ and the first rotational axis of $U_{1}$ in a same plane as in Fig.17(a), twists $\boldsymbol{S}_{11}, \boldsymbol{S}_{12}, \boldsymbol{S}_{13}$ are not independent with each other. Therefore, the rank of $\boldsymbol{S}_{1}$ degenerates into 4 and inverse kinematic singularity occurs.

Condition 2: As shown in Fig.17(b), when twists $S_{11}, S_{12}, S_{13}$ are parallel to the plane formed by twists $S_{14}$ and $\boldsymbol{S}_{15}$, the five elements in $S_{1}$ are not fully independent. Rank of $S_{1}$ is 4 and limb 1 is singular in this configuration. 


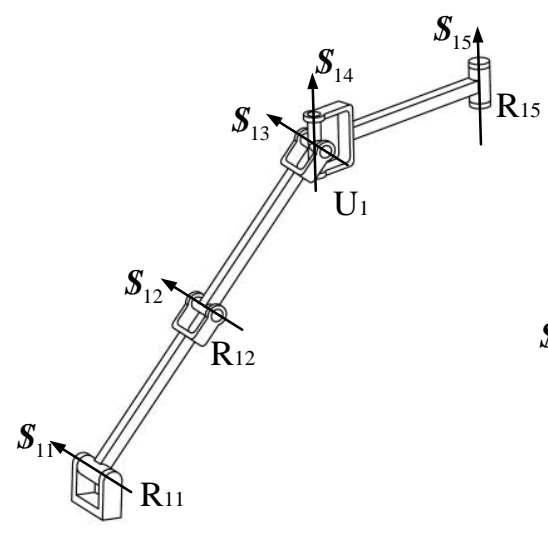

(a)

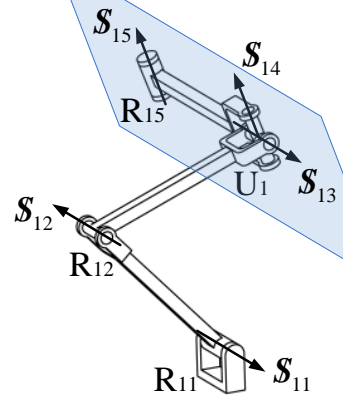

(b)

Fig.17 Two kinds of inverse kinematic singularities of limb 1: (a) condition 1; (b) condition 2

\subsubsection{Inverse kinematic singularity of limb 2 and limb 3}

For limb 2 and limb 3, the inverse kinematic singularities of the inner PMM should also be concerned. It is easy to know that the PMM locates at inverse kinematic singularities with link $A_{i 2} P_{i 1}$ coincident with link $P_{i 1} P_{i 2}$, one of those configurations is shown in Fig.18. Furthermore, the PMM is singular when all the links are coincident.

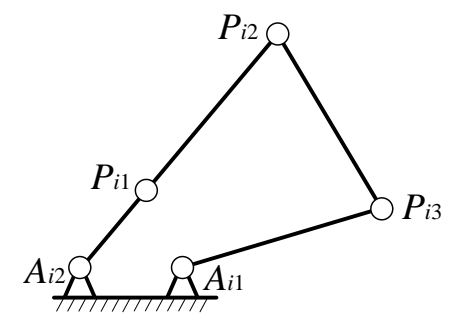

Fig.18 An inverse kinematic singular configuration of the PMM

When the PMM is free from inverse kinematic singularity, the twist system of limb $i(i=2,3)$ can be expressed as

$$
\boldsymbol{S}_{i}=\left[\begin{array}{llllll}
\boldsymbol{S}_{i 1}^{T} & \boldsymbol{S}_{i e}^{T} & \boldsymbol{S}_{i 3}^{T} & \boldsymbol{S}_{i 4}^{T} & \boldsymbol{S}_{i 5}^{T} & \boldsymbol{S}_{i 6}^{T}
\end{array}\right]^{T}(i=2,3)
$$

Limb $i$ will locate at inverse kinematic singularity configurations with the following three conditions satisfied.

Condition 3: vector $\mathbf{A}_{i 1} \mathbf{P}_{i 3}$ is parallel to the axes of twists $\boldsymbol{S}_{i 4}, \boldsymbol{S}_{i 5}$ and $\boldsymbol{S}_{i 6}$, as shown in Fig.19. In this configuration, twists $\boldsymbol{S}_{i 1}, \boldsymbol{S}_{i e}, \boldsymbol{S}_{i 4}, \boldsymbol{S}_{i 5}$ and $\boldsymbol{S}_{i 6}$ are not fully independent and $\boldsymbol{S}_{i}$ is degenerated.

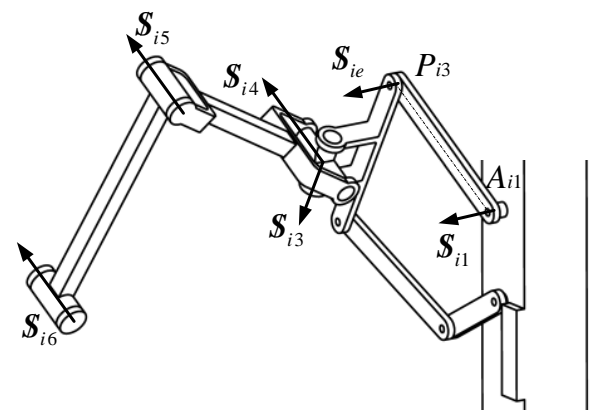

Fig.19 Inverse kinematic singularity corresponding to condition 3 
Condition 4: When limb $i$ moves to the configuration with axes of $\boldsymbol{S}_{i 4}, \boldsymbol{S}_{i 5}$ and $\boldsymbol{S}_{i 6}$ parallel to axes of $\boldsymbol{S}_{i 1}$ and $\boldsymbol{S}_{i e}$, only three of those five twists are independent. Twist system $S_{i}$ degenerates into a 4 -system and limb $i$ has only 4 DOFs in this condition.

Condition 5: Limb $i$ moves to the configuration with twists $\boldsymbol{S}_{i 4}, \boldsymbol{S}_{i 5}, \boldsymbol{S}_{i 6}$ in a same plane.

\subsection{Direct kinematic singularity}

The main reason for direct singularities is due to the linear dependence of wrench system consisting of constraint wrenches and actuation wrenches. The output link of a mechanism can have some infinitesimal motion in direct singular configurations. First of all, direct singularities of the PMM should be concerned. Due to its special dimensional parameters, the PMM will be direct singular only when all the links are coincident.

If the PMM is free from direct singularity, link $P_{i 2} P_{i 3}$ will be fixed with input joints $A_{i 1}$ and $A_{i 2}$ locked. In this condition, limb $i$ $(i=2,3)$ will exert a force wrench and a couple wrench on the moving platform. The couple wrench $\boldsymbol{S}_{\text {ric }}$ is perpendicular to the plane formed by the axes of joint $\mathrm{U}_{i}$, the force wrench $S_{r i F}$ locates at the center of joint $\mathrm{U}_{i}$ and is parallel to the axis of joint $\mathrm{R}_{i 6}$ $(i=2,3)$.

For limb 1 , there are two wrenches with input joint $\mathrm{R}_{11}$ locked. One is a constraint wrench denoted by $\boldsymbol{S}_{r 1 C}$, whose direction is defined by the normal of the plane formed by the two axes of universal joint $\mathrm{U}_{1}$, the other one denoted by $\boldsymbol{S}_{r 11}$ is an actuation force wrench that is reciprocal to screws $\boldsymbol{S}_{12}, \boldsymbol{S}_{13}, \boldsymbol{S}_{14}$ and $\boldsymbol{S}_{15}$. It is easy to know that $\boldsymbol{S}_{r 11}$ should be coincident with the intersecting line of two planes $\Sigma_{1}$ and $\Sigma_{2}$, one defined by $\boldsymbol{S}_{12}, \boldsymbol{S}_{13}$, the other one defined by $\boldsymbol{S}_{14}, \boldsymbol{S}_{15}$, as in Fig.20.

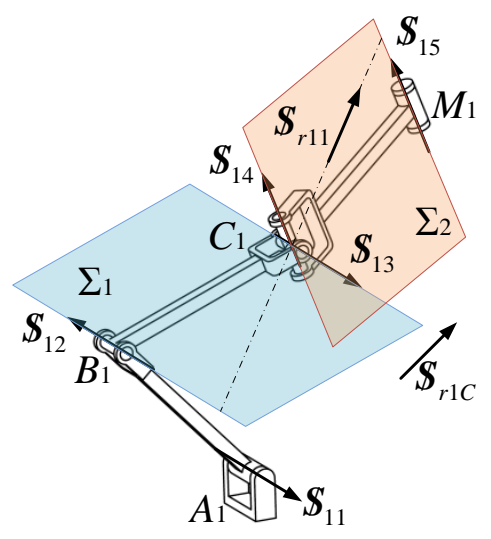

Fig.20 Constraint and actuation wrenches in limb 1

Therefore, when all the input joints are locked, six wrenches will be exerted on the moving platform as in Fig.21. These wrenches form a system $\boldsymbol{S}_{r}=\left[\begin{array}{llllll}\boldsymbol{S}_{r 1 C}^{T} & \boldsymbol{S}_{r 11}^{T} & \boldsymbol{S}_{r 2 C}^{T} & \boldsymbol{S}_{r 2 F}^{T} & \boldsymbol{S}_{r 3 C}^{T} & \boldsymbol{S}_{r 3 F}^{T}\end{array}\right]^{T}$, which will restrict all the six DOFs of the moving platform. However, if $S_{r}$ is not fully independent, direct singularity occurs and the moving platform gains one or more degrees of freedom. There are various conditions resulting in rank degeneration of $\boldsymbol{S}_{r}$. For example, when the mechanism working in 3T 


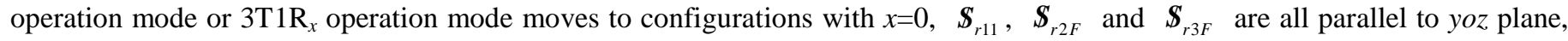
only two of those three wrenches are independent. $\boldsymbol{S}_{r}$ degenerates into a 5-system and the translational DOF along axis $x$ of the moving platform is out of control. Direct singular configurations can be identified by checking whether the rank of $\boldsymbol{S}_{r}$ is 6 or less than 6.

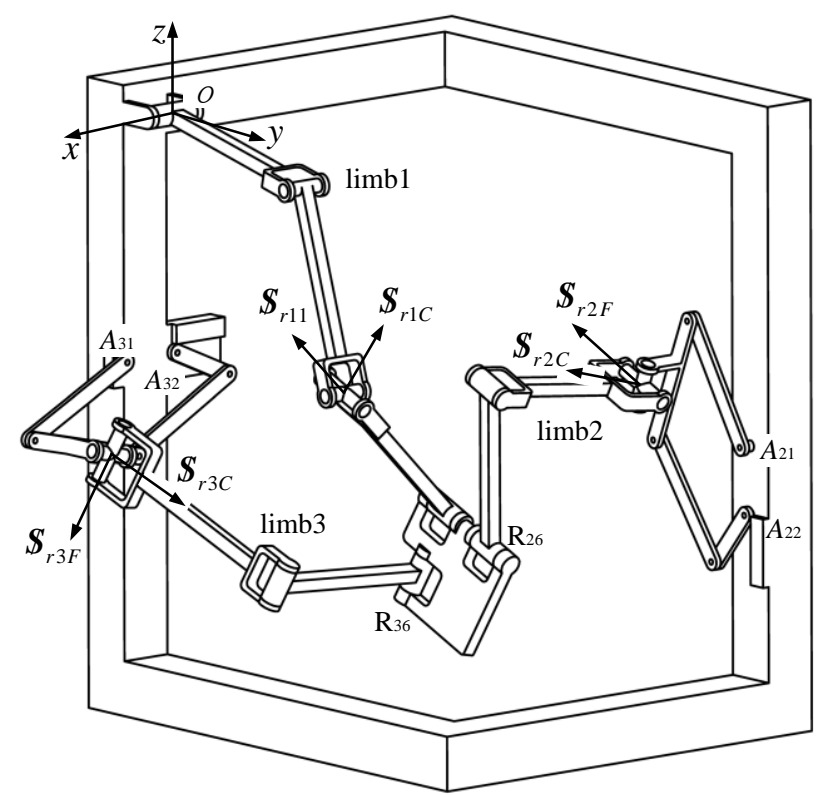

Fig.21 Constraint and actuation wrenches in the RPM

\section{Workspace evaluation and experiments}

In order to identify workspaces of the proposed RPM in different operation modes, the rotational angle limitations of passive joints as well as those of the input joints should be taken into consideration. In view of the practical joint architectures and to keep the mechanism away from singularities, rotational angle limitations of joints are given in Table.2. Fig.22 and Fig.23 show the angles in Table. 2 in detail, and the values of those angles can be calculated through inverse kinematic position analysis once the output parameters are given.

Table 2. Rotational range of angles

\begin{tabular}{llll}
\hline \hline Angle & Range & Angle & Range \\
\hline$\theta_{11}$ & {$[-\pi / 2, \pi / 2]$} & $\theta_{i 1}$ & {$[-\pi / 6,5 \pi / 12]$} \\
$\theta_{12}$ & {$[\pi / 6,5 \pi / 6]$} & $\theta_{i 2}$ & {$[\pi / 4, \pi]$} \\
$\theta_{13}$ & {$[\pi / 4,3 \pi / 4]$} & $\theta_{i 3}$ & {$[-\pi / 4, \pi / 4]$} \\
$\theta_{14}$ & {$[-\pi / 2, \pi / 2]$} & $\theta_{i 4}$ & {$[0, \pi]$} \\
$\psi_{i j}$ & {$[\pi / 12,11 \pi / 12]$} & $\theta_{i 5}(i=1,2 ; j=1,2,3)$ & {$[\pi / 6,5 \pi / 6]$} \\
\hline \hline
\end{tabular}




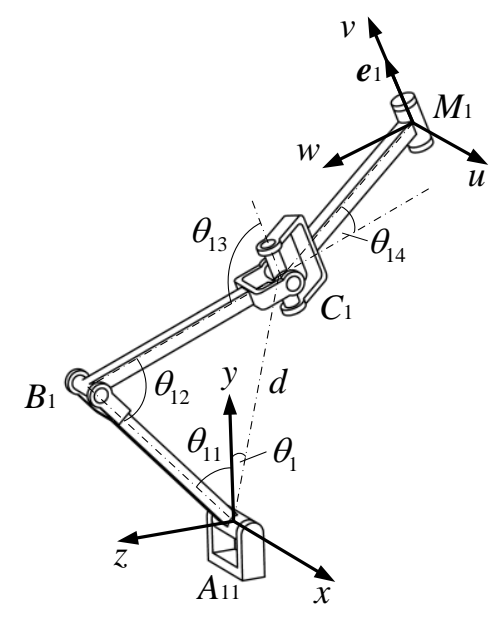

Fig.22 Angles in limb 1
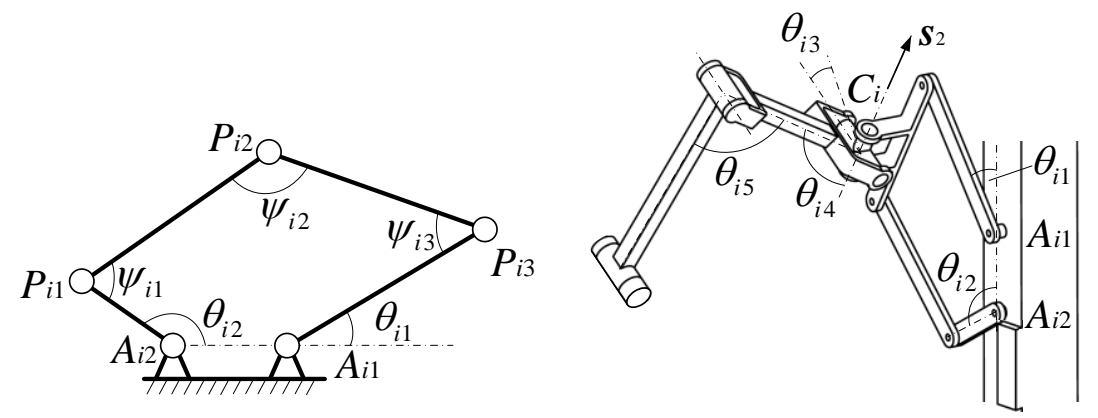

Fig.23 Angles in PMM and limb $i(i=2,3)$

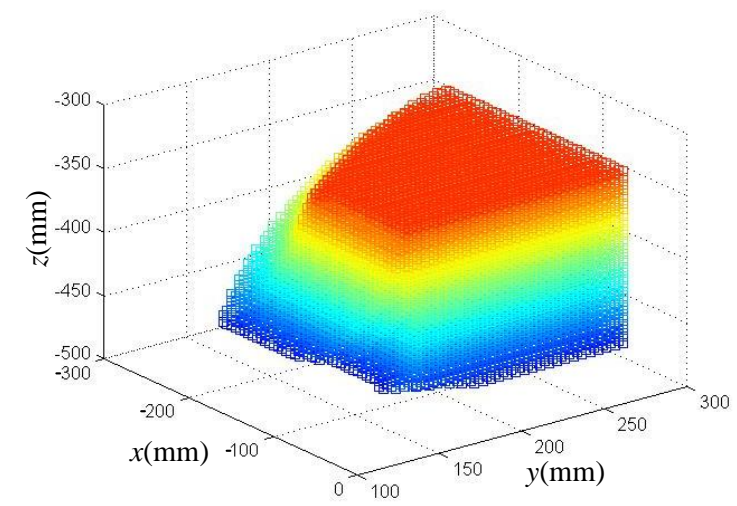

(a)

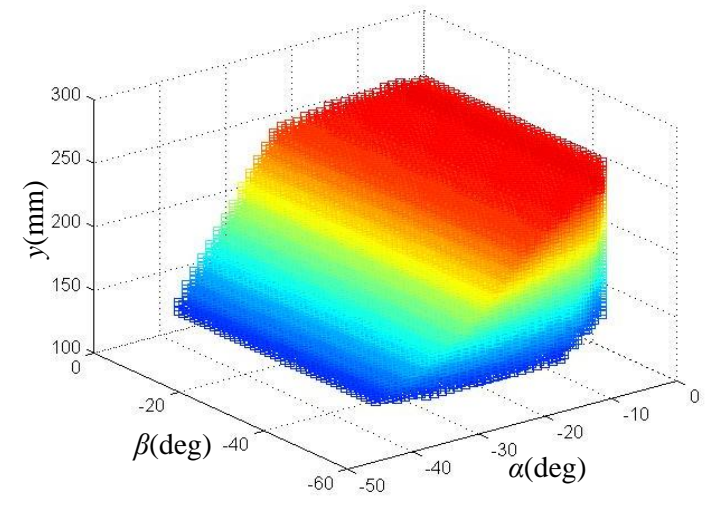

(b)

Fig.24 Workspaces in different operation modes: (a) in 3T operation mode; (b) in 3T2R operation mode

Through discretizing the possible workspace region and searching for those satisfy the limitations listed in Table.2, the workspaces of the RPM in different operation modes with architectural parameters given in Table.1 can be numerically determined. Fig.24 (a) shows the workspace in 3T operation mode, while Fig.24 (b) shows the one in 3T2R operation mode with $x=-149 \mathrm{~mm}$ and $z=-426 \mathrm{~mm}$. By slicing the workspace into several layers and analyzing those layers, one can find that the range of 
rotational angel around axis $x$ is $-40 \mathrm{deg} \sim 0 \mathrm{deg}$ and that around axis $y$ is $-60 \mathrm{deg} \sim 0 \mathrm{deg}$, which demonstrate the RPM has relative high rotational capability.

To verify the feasibility of the proposed mechanism for multidirectional AM, a prototype is developed as in Fig.25(a). A filament extruder of the fused deposition modeling process (a special kind of AM process) is fixed on the moving platform. Five serve motors are installed on input joints. A multi-axis motion controller is employed to control the motion of motors. With this prototype, multidirectional AM experiments on different surfaces are carried out. Fig.25(b) shows the AM process on a cylindrical surface, during which the RPM is working in $3 \mathrm{~T}_{1} \mathrm{R}_{x}$ operation mode with four driving motors. The manufacturing result is given in Fig.25(c). Experiments performed on the prototype demonstrate that the designed mechanism can be well applied in multidirectional AM process.

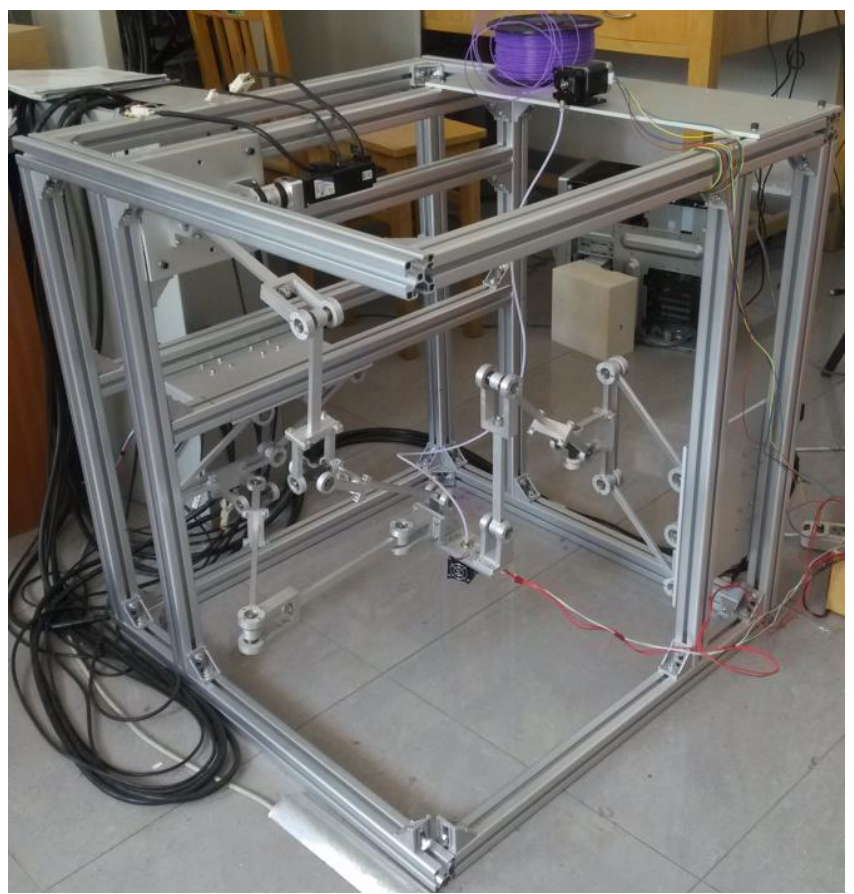

(a)

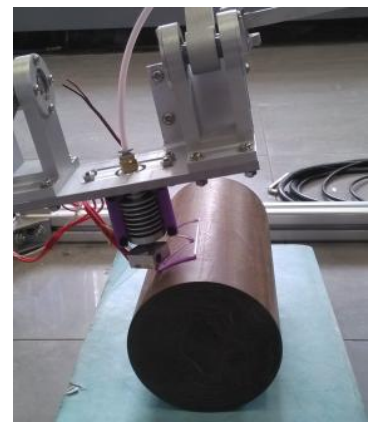

(b)

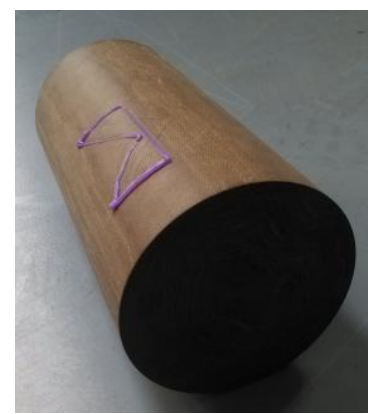

(c)

Fig.25 RPM prototype and experiments: (a) the prototype; (b) adding materials on a cylindrical surface; (c) the manufacturing result

\section{Conclusions}

In this paper, a novel RPM is proposed for multidirectional AM process. The RPM has three limbs, one C-limb and two hybrid limbs. The hybrid limbs are reconfigurable due to the existence of PMMs that have two working phases. By altering the PMMs into different phases, the RPM can reconfigure itself into four operation modes, i.e. $3 \mathrm{~T}$ operation mode, $3 \mathrm{~T} 1 \mathrm{R}_{x}$ operation mode, $3 \mathrm{~T}_{y}$ operation mode and $3 \mathrm{~T} 2 \mathrm{R}$ operation mode. Unified model for kinematic position analysis of the RPM is established. Numerical results of direct kinematic position solution and simulation results are compared, which demonstrate that the procedure for solving direct position solution is reliable. The overall Jacobian matrix is derived and singularity analysis is conducted. 
Workspaces evaluation shows that the RPM has relative high rotational capability. Multidirectional AM experiment of adding materials on a cylindrical surface is carried out, demonstrating that the proposed RPM can be well applied in multidirectional AM process.

\section{Acknowledgments}

The authors gratefully acknowledge the financial support of National Science Foundation of China under Grant No.51175029, 51475035, Beijing Natural Science Foundation under Grant No. 3132019 and the Program for New Century Excellent Talents in University of China (Grant No. NCET-12-0769).

\section{References}

[1] Fisher R, Podhorodeski R P, Nokleby S B. Design of a reconfigurable planar parallel manipulator. Journal of Robotic Systems, 2004, 21(12): 665-675.

[2] Xi F, Xu Y, Xiong G. Design and analysis of a re-configurable parallel robot. Mechanism and machine theory, 2006, 41(2): 191-211.

[3] Gogu G. Branching singularities in kinematotropic parallel mechanisms//Computational Kinematics. Springer Berlin Heidelberg, 2009: 341-348.

[4] Gogu G. Structural synthesis of parallel robots. Dordrecht: Springer, 2008.

[5] Ye W, Fang Y, Zhang K, et al. A new family of reconfigurable parallel mechanisms with diamond kinematotropic chain. Mechanism and Machine Theory, 2014, 74: 1-9.

[6] Zlatanov D, Bonev I, Gosselin C M. Constraint singularities as configuration space singularities. In: Advances in Robot Kinematics. Dordrecht: Kluwer Academic Press, 2002. 183-192

[7] Zlatanov D, Bonev I A, Gosselin C M. Constraint singularities of parallel mechanisms//Robotics and Automation, 2002. Proceedings. ICRA02. IEEE International Conference on. IEEE, 2002, 1: 496-502.

[8] Fanghella P, Galletti C, Giannotti E. Parallel robots that change their group of motion//Advances in Robot Kinematics. Springer Netherlands, 2006: 49-56.

[9] Kong X, Gosselin C M, Richard P L. Type synthesis of parallel mechanisms with multiple operation modes. Journal of Mechanical Design, 2007, 129(6): 595-601.

[10] Ruggiu M, Kong X. Mobility and kinematic analysis of a parallel mechanism with both PPR and planar operation modes. Mechanism and machine theory, 2012, 55: 77-90. 
[11] Li Q, Hervé J M. Parallel mechanisms with bifurcation of Schoenflies motion. Robotics, IEEE Transactions on, 2009, 25(1): 158-164

[12] Gan D, Dai J S, Dias J, et al. Unified kinematics and singularity analysis of a metamorphic parallel mechanism with bifurcated motion. Journal of Mechanisms and Robotics, 2013, 5(3): 031004.

[13] Gan D, Dai J S, Dias J, et al. Reconfigurability and unified kinematics modeling of a 3rTPS metamorphic parallel mechanism with perpendicular constraint screws[J]. Robotics and Computer-Integrated Manufacturing, 2013, 29(4): 121-128.

[14] Gan D, Dai J S, Liao Q. Mobility change in two types of metamorphic parallel mechanisms[J]. Journal of Mechanisms and Robotics, 2009, 1(4): 041007.

[15] Zhang K, Dai J S, Fang Y. Geometric constraint and mobility variation of two 3SvPSv metamorphic parallel mechanisms. Journal of Mechanical Design, 2013, 135(1): 011001.

[16] Zhang K, Dai J S. Screw-System-Variation Enabled Reconfiguration of the Bennett Plano-Spherical Hybrid Linkage and Its Evolved Parallel Mechanism. Journal of Mechanical Design, 2015, 137(6): 062303.

[17] Carbonari L, Callegari M, Palmieri G, et al. A new class of reconfigurable parallel kinematic machines[J]. Mechanism and Machine Theory, 2014, 79: 173-183.

[18] Palpacelli M C, Carbonari L, Palmieri G. Details on the Design of a Lockable Spherical Joint for Robotic Applications[J]. Journal of Intelligent \& Robotic Systems, 1-11.

[19] Palpacelli M C, Carbonari L, Palmieri G, et al. Analysis and Design of a Reconfigurable 3-DoF Parallel Manipulator for Multimodal Tasks[J]. IEEE/ASME Transactions on mechatronics, 2015.

[20] Carbonari L, Callegari M, Palmieri G, et al. Analysis of kinematics and reconfigurability of a spherical parallel manipulator[J]. Robotics, IEEE Transactions on, 2014, 30(6): 1541-1547.

[21] Coppola G, Zhang D, Liu K. A 6-DOF reconfigurable hybrid parallel manipulator. Robotics and Computer-Integrated Manufacturing, 2014, 30(2): 99-106.

[22] Zhang D, Bi Z. Development of reconfigurable parallel kinematic machines using modular design approach. Proceedings of the Canadian Engineering Education Association, 2011.

[23] Brisan C, Csiszar A. Computation and analysis of the workspace of a reconfigurable parallel robotic system. Mechanism and Machine Theory, 2011, 46(11): 1647-1668.

[24] Moosavian A, Xi F J. Design and analysis of reconfigurable parallel robots with enhanced stiffness. Mechanism and Machine Theory, 2014, 77: 92-110.

[25] Chen C T. Reconfiguration of a parallel kinematic manipulator for the maximum dynamic load-carrying capacity. Mechanism and Machine Theory, 2012, 54: 62-75. 
[26] Yoon J, Ryu J, Lim K B. Reconfigurable ankle rehabilitation robot for various exercises. Journal of Robotic Systems, 2006, 22(S1): S15-S33.

[27] Sabourin E, Houser S A, Helge Bøhn J. Accurate exterior, fast interior layered manufacturing. Rapid Prototyping Journal, 1997, 3(2): 44-52.

[28] Onuh S O, Hon K K B. Optimising build parameters for improved surface finish in stereo lithography. International Journal of Machine Tools and Manufacture, 1998, 38(4): 329-342.

[29] Lee W, Wei C, Chung S C. Development of a hybrid rapid prototyping system using low-cost fused deposition modeling and five-axis machining. Journal of Materials Processing Technology, 2014, 214(11): 2366-2374.

[30] Dwivedi R, Kovacevic R. An expert system for generation of machine inputs for laser-based multi-directional metal deposition. International Journal of Machine Tools and Manufacture, 2006, 46(14): 1811-1822.

[31] Dutta, B, Singh, V, Natu, H, et al. Direct Metal Deposition: Six-axis Direct Metal Deposition Technology Enables Creation/Coating of New Parts or Remanufacturing of Damaged Parts With Near Net-shape. Advanced Materials\& Processes, 2009, 167(3): 29-31.

[32] Dutta B, Palaniswamy S, Choi J, et al. Additive manufacturing by direct metal deposition. Advanced Materials \& Processes, 2011, 169(5): 33-36.

[33] Chen Y, Zhou C, Lao J. A layerless additive manufacturing process based on CNC accumulation. Rapid Prototyping Journal, 2011, 17(3): 218-227.

[34] Boisselier D, Sankaré S, Engel T. Improvement of the Laser Direct Metal Deposition Process in 5-axis Configuration[J]. Physics Procedia, 2014, 56:239-249.

[35] Horvath J. The Desktop 3D Printer[M]//Mastering 3D Printing. Apress, 2014: 11-20.

[36] Song X, Pan Y, Chen Y. Development of a Low-Cost Parallel Kinematic Machine for Multidirectional Additive Manufacturing. Journal of Manufacturing Science and Engineering, 2015, 137(2): 021005.

[37] Fang Y, Tsai L W. Structure synthesis of a class of 4-DoF and 5-DoF parallel manipulators with identical limb structures. The International Journal of Robotics Research, 2002, 21(9): 799-810.

[38] Ye W, Fang Y F, Guo S. Reconfigurable parallel mechanisms with planar five-bar metamorphic linkages. Science China Technological Sciences, 2014, 57(1): 210-218.

[39] Dai J S, Jones J R. Interrelationship between screw systems and corresponding reciprocal systems and applications[J]. Mechanism and machine theory, 2001, 36(5): 633-651.

[40] Dai J S, Jones J R. Mobility in metamorphic mechanisms of foldable/erectable kinds[J]. Journal of mechanical design, 1999, 121(3): 375-382. 
[41] Joshi S A, Tsai L W. Jacobian analysis of limited-DOF parallel manipulators. Journal of mechanical design, 2002, 124(2): 254-258.

[42] Tsai L W. Robot analysis: the mechanics of serial and parallel manipulators. John Wiley \& Sons, 1999. 